\title{
Maximal linear spaces contained in the base loci of pencils of quadrics
}

\author{
Xiaoheng Wang
}

\begin{abstract}
The geometry of the Fano variety of maximal linear spaces contained in the base locus of a pencil of quadrics has been studied by algebraic geometers when the base field is algebraically closed. In this paper, we work over an arbitrary base field of characteristic not equal to 2 and show how these Fano varieties are related to the Jacobians of hyperelliptic curves. In particular, if $B$ is the base locus of a generic pencil of quadrics in $\mathbb{P}^{2 n+1}$ and $F$ is the Fano variety of $(n-1)$-planes contained in $B$, then $F$ is a component of a disconnected commutative algebraic group $G=\operatorname{Pic}^{0}(C) \dot{\cup} F \dot{\cup} \operatorname{Pic}^{1}(C) \dot{\cup} F^{\prime}$, where $C$ is the hyperelliptic curve defined by the discriminant form of the pencil. In the second half of this paper, we study regular pencils of quadrics, where the hyperelliptic curve defined by the discriminant may be singular.
\end{abstract}

\section{Introduction}

Let $k$ be a field of characteristic not 2 . Let $\mathcal{L}=\left\{x Q_{1}-y Q_{2} \mid[x, y] \in \mathbb{P}^{1}\right\}$ be a pencil of quadrics in $\mathbb{P}^{N-1}$ for $N \geqslant 3$, where $Q_{1}$ and $Q_{2}$ are two linearly independent quadrics defined over $k$. Let $B=Q_{1} \cap Q_{2}$ denote the base locus. In this paper, we study the general geometry of the variety $F$ of linear subspaces contained in $B$ of maximal dimension. Let $A_{1}$ and $A_{2}$ denote two Gram matrices of $Q_{1}$ and $Q_{2}$ with respect to the same choice of basis. Let $f(x)$ be the polynomial of degree at most $N$ defined by

$$
f(x)=\operatorname{disc}\left(x A_{1}-A_{2}\right)=(-1)^{N(N-1) / 2} \operatorname{det}\left(x A_{1}-A_{2}\right) .
$$

We shall assume that $f(x)$ splits completely over a separable closure $k^{s}$ of $k$.

The geometry depends very much on the parity of $N$ and how "singular" the pencil is. A pencil is generic if $\mathcal{L}$ has transverse intersection with the hypersurface of singular quadrics in $\mathbb{P}\left(H^{0}\left(\mathcal{O}_{\mathbb{P} N-1}(2)\right)\right)$. Equivalently, $\mathcal{L}$ contains precisely $N$ singular quadrics over $k^{s}$, all of which are simple cones. This is also equivalent to saying that $f(x)$ has degree at least $N-1$ with no repeated roots. Suppose that $\mathcal{L}$ is generic. Denote by $C$ the hyperelliptic curve with affine equation $y^{2}=f(x)$. The isomorphism type of $C$ over $k$ is independent of the choice of the basis used to obtain the Gram matrices $A_{1}$ and $A_{2}$.

When $N=2 n+1$ is odd, the dimension of maximal linear subspaces contained in $B$ is $n-1$. We assume that $k$ has at least $N+1$ elements, which implies that there is a rational non-degenerate

Received 25 May 2017, accepted in final form 27 May 2017.

2010 Mathematics Subject Classification 14H40, 14N20.

Keywords: pencils of quadrics, Jacobians of hyperelliptic curves, torsors.

This journal is (C) Foundation Compositio Mathematica 2018. This article is distributed with Open Access under the terms of the Creative Commons Attribution Non-Commercial License, which permits non-commercial reuse, distribution, and reproduction in any medium, provided that the original work is properly cited. For commercial re-use, please contact the Foundation Compositio Mathematica. 


\section{WANG}

quadric over $k$ in the pencil. After renaming, we may assume that $Q_{1}$ is non-degenerate and hence the genus of $C$ is $n$. It is well known from intersection theory that geometrically over $k^{s}$, there are $2^{2 n}$ such $(n-1)$-planes. The arithmetic theory over $k$ was studied in [BG14], where it was shown that $F$ is a torsor of $J[2]$, where $J$ denotes the Jacobian of $C$. Note that the isomorphism type of $C$ over $k$ depends on the choice of the two quadrics $Q_{1}$ and $Q_{2}$ in $\mathcal{L}$, while the group scheme $J[2]$ is independent of this choice.

When $N=2 n+2$ is even, the theory is richer. The dimension of maximal linear subspaces contained in $B$ is still $n-1$, and the genus of $C$ is still $n$. The rational function $x$ defines a degree 2 morphism $C \rightarrow \mathbb{P}^{1}$. Let $D_{0}$ denote the hyperelliptic class obtained from pulling back the hyperplane section on $\mathbb{P}^{1}$. It was proved by Desale and Ramanan [DR76], Reid [Rei72], and Donagi [Don80] that geometrically over $k^{s}$, the variety $F$ is isomorphic to the Jacobian $J$ of $C$. Gauthier had also studied this in [Gau54]. The arithmetic theory when $C$ has genus 1 is known and is used heavily in studying the 4-descent of elliptic curves. The genus 2 case was known to Cassels [Cas93]. The main result of this paper is that for arbitrary $n \geqslant 1$, the variety $F$ is a torsor of $J$ over $k$ and, moreover, that the following theorem holds.

Theorem 1.1. Let $G$ be the disconnected variety

$$
G=\operatorname{Pic}^{0}(C) \dot{\cup} F \dot{\cup} \operatorname{Pic}^{1}(C) \dot{\cup} F^{\prime},
$$

where $F^{\prime}$ is a copy of $F$. Then there is a commutative algebraic group structure on $G$ over $k$ such that

1. $G^{0}=\operatorname{Pic}^{0}(C)$ with component group $G / G^{0} \simeq \mathbb{Z} / 4$;

2. $F^{\prime}$ is isomorphic to $F$ as varieties via the inversion map $-1_{G}$;

3. the group law extends that on $H=\operatorname{Pic}(C) / \mathbb{Z} D_{0} \simeq \operatorname{Pic}^{0}(C) \dot{\cup} \operatorname{Pic}^{1}(C)$.

Moreover, we will show that this structure is unique once we impose one more condition. See Theorem 2.25 for the complete statement.

When $k$ is algebraically closed, Donagi put a group law on $F$ in [Don80]. In light of Theorem 1.1, to put a group law on $F$ amounts to choosing a point $X_{0}$ on $F(k)$ as the identity. The identity that Donagi picked is an element of $F[4]=\left\{X \in F \mid X+_{G} X+_{G} X+_{G} X=0\right\}$. By studying the variety $G$ and putting a canonical group structure on it, we no longer need to make this choice.

For arithmetic applications, it is of significance that Theorem 1.1 implies that as classes of torsors of $J$, we have $2[F]=\left[\operatorname{Pic}^{1}(C)\right]$ in $H^{1}(k, J)$. In particular, if $\operatorname{Pic}^{1}(C)(k) \neq \emptyset$, then $F$ is a torsor of $J$ of order dividing 2 and for any $\left[D_{1}\right] \in \operatorname{Pic}^{1}(C)(k)$, one has a lift of $[F]$ to a torsor of $J[2]$ by taking

$$
F[2]_{\left[D_{1}\right]}=\left\{X \in F \mid X+{ }_{G} X=\left[D_{1}\right]\right\} .
$$

When the class $\left[D_{1}\right]$ comes from a rational point $P$ on $C$, the lift $F[2]_{P}$ can also be described geometrically; see Examples 2.27 and 2.30.

When the curve $C$ has a rational Weierstrass point $P$, it turns out that all torsors of $J[2]$ arise from pencils of quadrics as some $F[2]_{P}$. This is the key geometric input in [BG13], where Bhargava and Gross obtained the average sizes of the 2-Selmer groups of Jacobians of hyperelliptic curve with a rational Weierstrass point. When the curve $C$ only has a rational non-Weierstrass point $P$, not all torsors of $J[2]$ arise this way in general. However, when $k$ is a global field, all of the locally soluble torsors of $J[2]$, namely ones that correspond to elements of the 2-Selmer group, arise from pencils of quadrics. This is used in [SW18], where Shankar and the author obtained the average 


\section{PENCILS OF QUADRICS AND HYPERELLIPTIC CURVES}

sizes of the 2-Selmer groups of Jacobians of hyperelliptic curves with rational non-Weierstrass points.

In general, when restricted to $F$, the multiplication-by-2 map on $G$ gives an unramified map from $F$ to $\operatorname{Pic}^{1}(C)$ of degree $2^{2 n}$. Such a map is a twist of the multiplication-by-2 map from $\operatorname{Pic}^{0}(C)$ to itself and is called a two-cover of $\operatorname{Pic}^{1}(C)$. When $k=\mathbb{Q}$ is a global field and the curve $C$ has points locally everywhere, all locally soluble two-covers of $\operatorname{Pic}^{1}(C)$ arise this way. By counting these locally soluble two-covers of $\operatorname{Pic}^{1}(C)$ and comparing them to locally soluble twocovers of $\operatorname{Pic}^{0}(C)$, Bhargava, Gross, and the author [BGW17] showed that $\operatorname{Pic}^{0}(C)$ and $\operatorname{Pic}^{1}(C)$ are non-isomorphic a positive proportion of the time and so the curve $C$ has no points over any odd-degree extension of $\mathbb{Q}$ a positive proportion of the time.

In the second half of the paper, we generalize these results to the case where the pencil $\mathcal{L}$ is "slightly singular," or regular. A pencil $\mathcal{L}$ is regular if it only has simple cones as singular members. In other words, $\mathcal{L}$ could intersect tangentially to the discriminant hypersurface in $\mathbb{P}\left(H^{0}\left(\mathcal{O}_{\mathbb{P}^{N-1}}(2)\right)\right)$ but does not contain any quadrics with higher degeneracy degree than simple cones. Let $U$ denote the underlying $k$-vector space of dimension $N$, and view the two generating quadrics $Q_{1}, Q_{2}$ as linear operators $A_{1}, A_{2}: U \rightarrow U^{\vee}$. When $Q_{1}$ is smooth, $A_{1}$ is an isomorphism and the composite

$$
T: U \stackrel{A_{2}}{\longrightarrow} U^{\vee} \stackrel{A_{1}^{-1}}{\longrightarrow} U
$$

is self-adjoint with respect to $A_{1}$. We call $T$ the self-adjoint operator associated to the pencil. The pencil spanned by $Q_{1}$ and $Q_{2}$ is regular if and only if $T$ is regular, which by definition means that all the eigenspaces of $T$ are 1-dimensional. Note that the pencil is generic if and only if $T$ is regular semi-simple.

Suppose that $\mathcal{L}$ is regular with $Q_{1}$ non-degenerate and that $N=2 n+1$ is odd. Factor $f(x)$ as $f(x)=c \prod_{i=1}^{r+1}\left(x-\alpha_{i}\right)^{m_{i}}$ over $k^{s}$. Let $U_{i, T}$ denote the generalized eigenspace over $k^{s}$ of $T$ with eigenvalue $\alpha_{i}$. Since $T$ is self-adjoint with respect to $A_{1}$, its generalized eigenspaces are pairwise orthogonal. A projective $(n-1)$-plane contained in the base locus $B$ can be viewed as a linear $n$-plane $X$ such that $X \subset X^{\perp}$ and $T X \subset X^{\perp}$, where $\perp$ is taken with respect to $A_{1}$. For each $i=1, \ldots, r+1$, we define $\operatorname{dim}_{i, T}(X)$ to be the dimension of the maximal $T$-stable subspace of $\left(X \otimes k^{s}\right) \cap U_{i, T}$. Since each $U_{i, T}$ is $m_{i}$-dimensional and $A_{1}$ restricts to a non-degenerate quadratic form on $U_{i, T}$, we have

$$
\operatorname{dim}_{i, T}(X) \leqslant m_{i} / 2 .
$$

For any sequence of integers $d_{1}, \ldots, d_{r+1}$ such that $0 \leqslant d_{i} \leqslant m_{i} / 2$, we define

$$
L_{\left\{d_{1}, \ldots, d_{r+1}\right\}}^{T}\left(k^{s}\right)=\left\{X \simeq\left(k^{s}\right)^{n} \mid X \subset X^{\perp}, T X \subset X^{\perp}, \operatorname{dim}_{i, T}(X)=d_{i}\right\} .
$$

Note that the singular locus of $B$ consists of the projectivization of the eigenspaces of $T$ whose associated eigenvalues have multiplicity at least 2. Hence $L_{\{0, \ldots, 0\}}^{T}\left(k^{s}\right)$ is the set of $k^{s}$-points of the variety of projective $(n-1)$-planes contained in the smooth locus of $B$. We have the following counting result on the size of $L_{\left\{d_{1}, \ldots, d_{r+1}\right\}}^{T}\left(k^{s}\right)$.

Theorem 1.2. Let a denote the number of $d_{i}$ equal to $m_{i} / 2$. Then

$$
\left|L_{\left\{d_{1}, \ldots, d_{r+1}\right\}}^{T}\left(k^{s}\right)\right|=2^{r} / 2^{a} .
$$

Now, for any field $k^{\prime}$ containing $k$, one defines $L_{\left\{d_{1}, \ldots, d_{r+1}\right\}}^{T}\left(k^{\prime}\right)$ similarly. Let $J=\operatorname{Pic}^{0}(C)$ denote the generalized Jacobian of the (possibly singular) complete curve $C$ defined by the affine equation $y^{2}=f(x)$. 


\section{WANG}

Theorem 1.3. For any field $k^{\prime}$ containing $k$, the group $J[2]\left(k^{\prime}\right)$ acts on $L_{\left\{d_{1}, \ldots, d_{r+1}\right\}}^{T}\left(k^{\prime}\right)$ simply transitively if $a=0$ and transitively if $a>0$.

As before, the case when $N=2 n+2$ is even is more interesting. In what follows, we write $X$ for a linear subspace of $U$ and write $\mathbb{P} X$ for its projectivization. For any $v \in U \otimes k^{s}$, denote by $[v]$ the point of $\mathbb{P}(U)\left(k^{s}\right)$ corresponding to the line spanned by $v$. As before, let $C$ denote the hyperelliptic curve defined by the affine equation $y^{2}=f(x)$. Then $C$ is smooth if and only if the pencil is generic. When all the roots of $f$ have multiplicity at most 2 , the curve $C$ is nodal. Denote by $p_{g}$ the geometric genus of $C$, defined as the genus of the normalization $\widetilde{C}$. As we will see in Proposition 3.12, when $p_{g}=-1$, the curve $C$ is reducible and the base locus $B$ contains a unique $\mathbb{P}^{n}$. When $p_{g} \geqslant 0$, the base locus $B$ contains no $\mathbb{P}^{n}$ and we define

$$
F_{0}=\{\mathbb{P} X \mid \operatorname{dim} \mathbb{P} X=n-1, \mathbb{P} X \subset B\} .
$$

As in the odd case, we need to impose certain open conditions to obtain interesting relations with the Jacobian of $C$. Consider

$$
F=\left\{\mathbb{P} X \in F_{0} \mid[v] \notin \mathbb{P} X \text { for all singular points }[v] \in B\right\} .
$$

Bhosle [Bho90] proved that when $C$ only has nodal singularities, $F$ is isomorphic to the generalized Jacobian of $C$ over $k^{s}$. Over the base field $k$, we have the following result.

TheOREm 1.4. Suppose $p_{g} \geqslant 0$ and that $C$ only has nodal singularities. Let $G$ be the disconnected variety

$$
G=\operatorname{Pic}^{0}(C) \dot{\cup} F \dot{\cup} \operatorname{Pic}^{1}(C) \dot{\cup} F^{\prime},
$$

where $F^{\prime}$ is a copy of $F$. There is a commutative algebraic group structure on $G$ over $k$ such that

1. $G^{0}=\operatorname{Pic}^{0}(C)$ with component group $G / G^{0} \simeq \mathbb{Z} / 4$;

2. $F^{\prime}$ is isomorphic to $F$ as varieties via the inversion map $-1_{G}$;

3. the group law extends that on $H=\operatorname{Pic}(C) / \mathbb{Z} D_{0} \simeq \operatorname{Pic}^{0}(C) \dot{\cup} \operatorname{Pic}^{1}(C)$, where $D_{0}$ is the hyperelliptic class.

Corollary 1.5. Over $k^{s}$, one can take $F_{0}$ as a compactification of the generalized Jacobian $\operatorname{Pic}^{0}(C)$.

We expect Theorem 1.4 to be true without the nodal condition on $C$. Let $\widetilde{F}$ denote the torsor of $J(\widetilde{C})$ obtained from certain reductions (see Section 3) on the pencil $\mathcal{L}$ to the generic case. Without the condition on $C$, we can prove the following result.

Theorem 1.6. Suppose $p_{g} \geqslant 0$. Then there is a surjection $F \rightarrow \widetilde{F}$. Over $k^{s}$, the pre-image of every point has a filtration with $\mathbb{G}_{a^{-}}$and $\mathbb{G}_{m}$-factors. The kernel of the natural map $J(C) \rightarrow J(\widetilde{C})$ has a filtration with the same factors.

The theory of regular pencils is used in [BGW17] for the study of two-covers of $\operatorname{Pic}^{1}(C)$. As noted by Poonen and Schaefer in [PS97, footnote 2], it is not always enough to study only unramified covers of the hyperelliptic curve $C$ when studying 2-descent on its Jacobian when $C$ has no rational Weierstrass points; one also needs covers of $C$ unramified away from the two points above some fixed point on $\mathbb{P}^{1}$. Identifying these two points gives a nodal curve, and regular pencils help in understanding two-covers for the generalized Jacobian of this nodal curve.

The paper is organized as follows. In Section 2, we focus on the case of generic pencils. We first recall from [BG14] the results in the case when $N$ is odd. We then study the case when $N$ 


\section{PENCILS OF QUADRICS AND HYPERELLIPTIC CURVES}

is even and prove Theorem 1.1. We finish with examples of special pencils in relation to the applications in [BG13] and [SW18]. In Section 3, we perform the parallel analysis in the case of regular pencils and prove Theorems 1.2-1.6.

\section{Generic pencils}

Let $k$ be a field of characteristic not 2. Let $Q_{1}$ and $Q_{2}$ be two linearly independent quadratic forms on a $k$-vector space $U$ of dimension $N$. In this section, we study the general geometry of the maximal isotropic subspaces with respect to both quadrics.

There are three equivalent ways to formulate this problem. We call the above formulation the $\left(Q_{1}, Q_{2}\right)$-setup. Now, suppose that $Q_{1}$ is non-degenerate. Let $b_{1}$ and $b_{2}$ denote the bilinear forms corresponding to $Q_{1}$ and $Q_{2}$, respectively. More explicitly,

$$
b_{i}(v, w)=\frac{1}{2}\left(Q_{i}(v+w)-Q_{i}(v)-Q_{i}(w)\right) .
$$

Let $T: U \rightarrow U$ be the unique operator such that for all $v, w \in U$,

$$
b_{2}(v, w)=b_{1}(v, T w) .
$$

Note that $T$ is self-adjoint with respect to $b_{1}$ since $b_{1}$ and $b_{2}$ are symmetric.

To say that a linear subspace $X$ is isotropic with respect to both $Q_{1}$ and $Q_{2}$ is the same as saying

$$
X \subset X^{\perp_{Q_{1}}}, \quad T X \subset X^{\perp_{Q_{1}}} .
$$

Therefore, instead of starting with a pair of quadratic forms, we could have started with a nondegenerate quadratic form along with a self-adjoint operator. We call this formulation the $\left(Q_{1}, T\right)$ setup.

Lastly, we could view $Q_{1}$ and $Q_{2}$ as quadrics in $\mathbb{P}(U)$ and take a pencil $\mathcal{L}=\left\{x Q_{1}-y Q_{2} \mid\right.$ $\left.[x, y] \in \mathbb{P}^{1}\right\}$ of quadrics in $\mathbb{P}(U)$. Let $B=Q_{1} \cap Q_{2}$ denote the base locus. The above problem regarding common isotropic subspaces translates into studying the Fano variety of maximal linear subspaces contained in the base locus. We call this formulation the $(\mathbb{P}(U), \mathcal{L})$-setup.

We define the notion of generic in each of the three formulations. With the $\left(Q_{1}, Q_{2}\right)$-setup, we require $f(x)=(-1)^{N(N-1) / 2} \operatorname{det}\left(x A_{1}-A_{2}\right)$ to have no repeated roots, where $A_{1}$ and $A_{2}$ are Gram matrices for $Q_{1}$ and $Q_{2}$ with respect to the same choice of basis. With the $(Q, T)$-setup, we require the characteristic polynomial $f_{T}(x)=\operatorname{det}(x I-T)$ of the self-adjoint operator $T$ to have no repeated roots. We will also assume that $k$ has at least $N+1$ elements, for otherwise there might not exist a rational non-degenerate $Q$ in the pencil. With the $(\mathbb{P}(U), \mathcal{L})$-setup, we require that the pencil $\mathcal{L}$ intersects the hypersurface of singular quadrics in $\mathbb{P}\left(H^{0}\left(\mathcal{O}_{\mathbb{P}^{N-1}}(2)\right)\right)$ transversely. Equivalently, $\mathcal{L}$ contains precisely $N$ singular quadrics over $k^{s}$, all of which are simple cones.

\subsection{Odd dimension}

We first consider the case when $U$ has dimension $N=2 n+1$. Suppose that $Q_{1}$ is non-degenerate. Let $C$ be the hyperelliptic curve of genus $n$ defined by the affine equation

$$
y^{2}=f(x)=(-1)^{n} \operatorname{det}\left(x A_{1}-A_{2}\right) .
$$

The isomorphism type of $C$ over $k$ is independent of the choice of the basis used for the Gram matrices $A_{1}$ and $A_{2}$. Let $J$ denote the Jacobian of $C$. Suppose that the pencil is generic and $f(x)$ 


\section{WANG}

splits completely over $k^{s}$. Let $Q=Q_{1}$, and let $T$ denote the corresponding self-adjoint operator. Let $W_{T}$ denote the $k$-scheme of (linear) $n$-dimensional planes $X \subset U$ such that $X \subset X^{\perp}$ and $T X \subset X^{\perp}$.

The geometry over $k^{s}$ is well known using classical intersection theory [Don80, Section 1.2]. We thank Elkies for the following explicit description of the $2^{2 n}$ elements of $W_{T}\left(k^{s}\right)$. After a change of basis over $k^{s}$, one can assume that the two quadrics are given by

$$
\begin{aligned}
& Q_{1}(x)=x_{1}^{2}+x_{2}^{2}+\cdots+x_{2 n+1}^{2} \\
& Q_{2}(x)=c_{1} x_{1}^{2}+c_{2} x_{2}^{2}+\cdots+c_{2 n+1} x_{2 n+1}^{2}
\end{aligned}
$$

for some pairwise distinct $c_{1}, \ldots, c_{2 n+1} \in k^{s}$. The following system of linear equations in $D_{1}, \ldots$, $D_{2 n+1}$ has a 1-dimensional kernel:

$$
\begin{aligned}
D_{1}+D_{2}+\cdots+D_{2 n+1} & =0 \\
c_{1} D_{1}+c_{2} D_{2}+\cdots+c_{2 n+1} D_{2 n+1} & =0 \\
& \vdots \\
c_{1}^{2 n-1} D_{1}+c_{2}^{2 n-1} D_{2}+\cdots+c_{2 n+1}^{2 n-1} D_{2 n+1} & =0 .
\end{aligned}
$$

Choose a basis $\left(D_{1}, \ldots, D_{2 n+1}\right)$ for the kernel and note that $D_{i} \neq 0$ for all $i$. For each $i=$ $1, \ldots, 2 n+1$, let $d_{i}$ be a square root of $D_{i}$. Any choice of a system of square roots gives an element of $W_{T}\left(k^{s}\right)$ by taking

$$
X=\left\{\left(d_{1} P\left(c_{1}\right), \ldots, d_{2 n+1} P\left(c_{2 n+1}\right)\right) \mid P \text { is any polynomial of degree at most } n-1\right\} .
$$

Note that if $\left(d_{1} P\left(c_{1}\right), \ldots, d_{2 n+1} P\left(c_{2 n+1}\right)\right)=\left(d_{1}^{\prime} P^{\prime}\left(c_{1}\right), \ldots, d_{2 n+1}^{\prime} P^{\prime}\left(c_{2 n+1}\right)\right)$, where $d_{i}= \pm d_{i}^{\prime}$ for all $i=1, \ldots, 2 n+1$ and $P$ and $P^{\prime}$ are polynomials of degree at most $n-1$, then either $P+P^{\prime}$ or $P-P^{\prime}$ vanishes on at least $n+1$ elements out of $c_{1}, \ldots, c_{2 n+1}$, implying that either $P=P^{\prime}$, in which case $d_{i}=d_{i}^{\prime}$ for all $i$, or $P=-P^{\prime}$, in which case $d_{i}=-d_{i}^{\prime}$ for all $i$. This shows that negating all of the $d_{i}$ is the only way to produce the same $n$-plane. Hence we obtain $2^{2 n}$ such $n$-planes from the different choices of square roots.

The arithmetic aspect of the theory has been studied by Bhargava and Gross in [BG14]; we list the results without proof. Consider the following two schemes over $k$ :

$$
\begin{gathered}
V_{f}=\{T: U \rightarrow U \mid T \text { is self-adjoint with respect to } Q \\
\quad \text { with characteristic polynomial } f\} \subset \mathbb{A}^{(2 n+1)^{2},}, \\
W_{f}=\left\{(T, X) \in V_{f} \times \operatorname{Gr}(n, U) \mid X \subset X^{\perp} \text { and } T X \subset X^{\perp}\right\} .
\end{gathered}
$$

Note that $W_{T}$ is the fiber of $W_{f}$ above a fixed $T$. The group $\operatorname{PO}(U, Q)=O(U, Q) /( \pm 1)$ acts on $V_{f}$ and $W_{f}$ via

$$
g \cdot T=g T g^{-1}, \quad g \cdot(T, X)=\left(g T g^{-1}, g X\right) .
$$

Proposition 2.1. Suppose that $f(x)$ splits completely in $k^{s}$ with no repeated roots. Then the action of $\mathrm{PO}(U, Q)$ on $V_{f}$ has a unique geometric orbit. For any $T \in V_{f}\left(k^{\prime}\right)$ defined over some field $k^{\prime}$ over $k$, its stabilizer scheme $\operatorname{Stab}(T)$ is isomorphic to $\operatorname{Res}_{L^{\prime} / k^{\prime}} \mu_{2} / \mu_{2} \simeq J[2]$ as group schemes over $k^{\prime}$, where $L^{\prime}=k^{\prime}[x] / f(x)$.

For general $Q$, there might not be a self-adjoint operator defined over $k$ with the prescribed characteristic polynomial. For example, over $\mathbb{R}$, operators self-adjoint with respect to a positivedefinite form have real eigenvalues. This is not a problem if $Q$ is split, that is, if there exists a linear space of dimension $n$ over $k$ isotropic with respect to $Q$. 


\section{PENCILS OF QUADRICS AND HYPERELLIPTIC CURVES}

Proposition 2.2. Suppose that $f(x)$ splits completely in $k^{s}$ with no repeated roots. If $Q$ is split over $k$, then $V_{f}(k)$ and $W_{f}(k)$ are non-empty. Furthermore, there exists $\left(T_{0}, X_{0}\right) \in W_{f}(k)$ with trivial stabilizer in $\mathrm{PO}(U, Q)\left(k^{a}\right)$, where $k^{a}$ denotes an algebraic closure of $k$.

THEOREM 2.3. Suppose that $k$ is separably closed of characteristic not 2, and suppose that $f(x)$ splits completely in $k^{s}$ with no repeated roots. Then $\mathrm{PO}(U, Q)(k)$ acts simply transitively on $W_{f}(k)$.

COROLlary 2.4. Suppose that $k$ is arbitrary of characteristic not 2, and suppose that $f(x)$ splits completely in $k^{s}$ with no repeated roots. If $W_{f}(k)$ is non-empty, then $\mathrm{PO}(U, Q)\left(k^{\prime}\right)$ acts simply transitively on $W_{f}\left(k^{\prime}\right)$ for any field $k^{\prime}$ over $k$.

COROLlary 2.5. Suppose that $k$ is arbitrary of characteristic not 2, and suppose that $f(x)$ splits completely in $k^{s}$ with no repeated roots. Let $T \in V_{f}(k)$, and let $J$ denote the Jacobian of the hyperelliptic curve defined by $y^{2}=f(x)$. Then $W_{T}$ is a torsor for $J[2]$.

Remark 2.6. We now write down an explicit formula for the identification

$$
J[2] \simeq \operatorname{Stab}(T) .
$$

We work over $k^{s}$, and it will be clear that the map is Galois-equivariant. Denote the roots of $f(x)$ over $k^{s}$ by $\alpha_{1}, \ldots, \alpha_{2 n+1}$, and the Weierstrass point corresponding to the root $\alpha_{i}$ by $P_{i}$ for $i=1, \ldots, 2 n+1$. Recall that $J[2]$ is an elementary 2-group generated by the $\left(P_{i}\right)-(\infty)$ with the only relation being that their sum is trivial. For each generator $\left(P_{i}\right)-(\infty)$, one looks for a polynomial $g_{i}(x)$ such that $g_{i}\left(\alpha_{i}\right)=-1$ and $g_{i}\left(\alpha_{j}\right)=1$ for all $j \neq i$. Then $g_{i}(T)$ is the image of $\left(P_{i}\right)-(\infty)$ in $\operatorname{Stab}(T)$. The image does not depend on the choice of the polynomial $g_{i}$ because any two choices differ by some multiples of $f(x)$ and $f(T)=0$. More explicitly, we define $h_{i}(x)=f(x) /\left(x-\alpha_{i}\right)$ and take

$$
g_{i}(x)=1-2 \frac{h_{i}(x)}{h_{i}\left(\alpha_{i}\right)} .
$$

In other words, on the level of $k^{s}$-points, (2.3) is given by

$$
\sum\left(\left(\alpha_{i}\right)-(\infty)\right) \mapsto \prod\left(1-2 \frac{h_{i}(T)}{h_{i}\left(\alpha_{i}\right)}\right)=1-2 \sum \frac{h_{i}(T)}{h_{i}\left(\alpha_{i}\right)}
$$

The summations and product are written without indices, meaning that the equality holds for any (finite) collection of matching indices.

See Remark 2.29 for a different viewpoint of (2.3).

\subsection{Even dimension}

Next, consider the case when $U$ has dimension $2 n+2$. The projective formulation is easier to work with in this case.

Let $\mathcal{L}=\left\{x Q_{1}-y Q_{2} \mid[x, y] \in \mathbb{P}^{1}\right\}$ be a rational generic pencil of quadrics in $\mathbb{P}^{2 n+1}=\mathbb{P}(U)$. Rationality means that it is generated by two quadrics $Q_{1}$ and $Q_{2}$ defined over $k$. The cone points of the $2 n+2$ singular quadrics are best understood in terms of the self-adjoint operator $T$ defined in (2.1), assuming that $Q_{1}$ is non-degenerate. The quadric $\lambda Q_{1}-Q_{2}$ is singular if and only if $\lambda$ is an eigenvalue of $T$. If we denote a corresponding eigenvector by $v_{\lambda}$, then the cone point of $\lambda Q_{1}-Q_{2}$ is $\left[v_{\lambda}\right] \in \mathbb{P}(U)$. In particular, the $2 n+2$ cone points span the entire $\mathbb{P}(U)$.

Since $\mathcal{L}$ is generic, the maximal (projective) dimension of any linear space contained in the 


\section{WANG}

base locus $B$ is $n-1$. Consider the following variety defined over $k$ :

$$
F=\{\mathbb{P} X \mid \operatorname{dim}(\mathbb{P} X)=n-1, \mathbb{P} X \subset B\} .
$$

The hyperelliptic curve $C$. For any rational generic pencil $\mathcal{L}$, there is an associated hyperelliptic curve defined as follows.

For any quadric $Q$ in $\mathbb{P}^{2 n+1}$, one defines its Lagrangian variety by

$$
L_{Q}=\{\mathbb{P} Y \mid \mathbb{P} Y \subset Q, \operatorname{dim}(\mathbb{P} Y)=n\} \subset \mathbb{G r}(n, \mathbb{P}(U)) .
$$

We recall some facts about $L_{Q}$; see also [Har92, Lecture 22]. When $Q$ is smooth, $L_{Q}$ has two connected components, also called the rulings of $n$-planes in $Q$. Two $n$-planes in $Q$ lie in the same ruling if and only if their intersection codimension is even. If $Q$ is defined over some field $k^{\prime}$, its discriminant is defined by

$$
\operatorname{disc}(Q)=(-1)^{n+1} \operatorname{det}(Q) k^{\prime \times 2} \in k^{\prime \times} / k^{\prime \times 2} .
$$

The connected components of $L_{Q}$ are defined over $k^{\prime}(\sqrt{\operatorname{disc}(Q)})$. In other words, $L_{Q}\left(k^{\prime s}\right)$ hits both rulings, and the $\operatorname{Gal}\left(k^{\prime s} / k^{\prime}(\sqrt{\operatorname{disc}(Q)})\right)$-action on $L_{Q}\left(k^{\prime s}\right)$ preserves the rulings. ${ }^{1}$ When $Q$ is singular, $L_{Q}$ has only one connected component.

Consider the variety

$$
\widetilde{F}=\left\{\left(x Q_{1}-y Q_{2}, \mathbb{P} Y\right) \mid[x, y] \in \mathbb{P}^{1}, \mathbb{P} Y \in L_{x Q_{1}-y Q_{2}}\right\} \subset \mathcal{L} \times \mathbb{G r}(n, \mathbb{P}(U)) .
$$

There is an obvious projection map $p_{1}: \widetilde{F} \rightarrow \mathbb{P}^{1}$. The fiber over $[x, y] \in \mathbb{P}^{1}$ is isomorphic to $L_{x Q_{1}-y Q_{2}}$. Let

$$
\epsilon: \tilde{F} \rightarrow C, \quad \pi: C \rightarrow \mathbb{P}^{1}
$$

denote the Stein factorization of $p_{1}$. In other words, $\epsilon$ has connected fibers, while the fibers of $\pi$ correspond bijectively to the connected components of the fibers of $p_{1}$. Hence $C$ is a double cover of $\mathbb{P}^{1}$ branched over the $2 n+2$ points that correspond to the singular quadrics on the pencil. A homogeneity analysis as in [Don80, Lemma 1.6] shows that $C$ is smooth at the ramification points. Hence $C$ is a hyperelliptic curve of genus $n$ parameterizing the rulings in the pencil. We call $C$ the hyperelliptic curve associated to the pencil. The Weierstrass points of $C$ correspond to the $2 n+2$ points on $\mathbb{P}^{1}$ cut out by the binary form $\operatorname{det}\left(x Q_{1}-y Q_{2}\right)$ of degree $2 n+2$. The curve $C$ is isomorphic over $k$ to the hyperelliptic curve defined by the affine equation

$$
y^{2}=(-1)^{n+1} \operatorname{det}\left(x Q_{1}-Q_{2}\right),
$$

canonically up to the hyperelliptic involution.

It was known to algebraic geometers [Rei72, DR76, Don80] that when $k$ is separably closed, $F$ is isomorphic to $J$, the Jacobian of the curve $C$ defined above. Therefore, it is natural to expect that over a general field, $F$ is a torsor of $J$.

\footnotetext{
${ }^{1}$ We give a proof of this fact. Let $Q$ be a non-degenerate quadric in $\mathbb{P}^{2 n+1}$ over $k^{\prime}$. Then by diagonalization, we may fix a basis $\left\{e_{1}, \ldots, e_{2 n+2}\right\}$ with respect to which $Q$ is given by the quadratic form $a_{1} x_{1}^{2}+\cdots+a_{2 n+2} x_{2 n+2}^{2}$. The Lagrangian variety $L_{Q}$ of $Q$ is the disjoint union of two irreducible components. An element in Gal $\left(k^{\prime s} / k^{\prime}\right)$ either fixes the components, or interchanges them. One may then take the isotropic (linear) $(n+1)$-plane $Y=\operatorname{Span}\left\{\left(1 / \sqrt{a_{1}}\right) e_{1}+\left(1 / \sqrt{-a_{2}}\right) e_{2}, \ldots,\left(1 / \sqrt{a_{2 n+1}}\right) e_{2 n+1}+\left(1 / \sqrt{-a_{2 n+2}}\right) e_{2 n+2}\right\}$ and observe that an element of $\operatorname{Gal}\left(k^{\prime s} / k^{\prime}\right)$ sends $Y$ to an element in the same ruling if and only if it fixes an even number of the square roots $\sqrt{a_{1}}, \sqrt{-a_{2}}, \ldots, \sqrt{a_{2 n+1}}, \sqrt{-a_{2 n+2}}$. The proof is now complete on noting that the discriminant of $Q$ is $(-1)^{n+1} a_{1} \cdots a_{2 n+2}=a_{1}\left(-a_{2}\right) \cdots a_{2 n+1}\left(-a_{2 n+2}\right)$.
} 


\section{PENCILS OF QUADRICS AND HYPERELLIPTIC CURVES}

A morphism $\tau: C \times F \rightarrow F$ that will serve as subtraction. Given any pair $(c, \mathbb{P} X) \in C \times F$, there is a unique $n$-plane $\mathbb{P} Y$ containing $\mathbb{P} X$ in the ruling of the quadric defined by $c$. Let $Q$ be another quadric in the pencil. Since the base locus contains no $n$-planes, $\mathbb{P} Y \cap B=\mathbb{P Y} \cap Q$ is a quadric in $\mathbb{P} Y$ containing $\mathbb{P} X$. Hence $\mathbb{P} Y \cap B=\mathbb{P} X \cup \mathbb{P} X^{\prime}$ is the union of two possibly equal $(n-1)$-planes. We define $\tau(c, \mathbb{P} X)$ to be $\mathbb{P} X^{\prime}$. For any $c \in C$, define the involution $\tau(c): F \rightarrow F$ by $\tau(c) \mathbb{P} X=\tau(c, \mathbb{P} X)$.

We can write down a more explicit formula for $\tau$ as follows. Given any $(c, \mathbb{P} X) \in C \times F$, let $\mathbb{P} Y$ denote the unique $n$-plane containing $\mathbb{P} X$ in the ruling of the quadric specified by $c$. Since $\mathbb{P} Y \nsubseteq Q$, there exists $p \in Y \backslash X$ such that $b(p, p) \neq 0$, where $b$ is the bilinear form associated to $Q$. There is a linear map on $U \otimes k^{s}$ given by reflection about $p^{\perp_{Q}}$, namely

$$
\operatorname{refl}_{p}: v \mapsto v-2 \frac{b(v, p)}{b(p, p)} p .
$$

Then $\operatorname{refl}_{p}(X) \subset Y$ is isotropic with respect to $Q$ and we have

$$
\tau(c, \mathbb{P} X)=\mathbb{P}\left(\operatorname{refl}_{p}(X)\right) .
$$

Our goal is to put a natural group structure on $G=\operatorname{Pic}^{0}(C) \dot{\cup} F \dot{\cup} \operatorname{Pic}^{1}(C) \dot{\cup} F^{\prime}$, where $F^{\prime}$ is a copy of $F$ as algebraic varieties. We do so by defining a simply transitive action of $H=\operatorname{Pic}(C) / \mathbb{Z} D_{0}$ on $F \dot{\cup} F^{\prime}$, for then we can define $+_{G}$ as follows: for any $x, x^{\prime} \in F \dot{\cup} F^{\prime}$, $[D],\left[D^{\prime}\right] \in H$,

1. $[D]+{ }_{G}\left[D^{\prime}\right]$ is the usual addition in $H$;

2. $x+{ }_{G}[D]=x+[D]$ is the image of $x$ under the action of $[D]$;

3. $x+{ }_{G} x^{\prime}$ is the unique element in $H$ that sends $-x^{\prime}$ to $x$.

An action of $\operatorname{Div}(C)$ on $F \dot{\cup} F^{\prime}$. For any $x \in F$, write suggestively $-x$ for the corresponding element of $F^{\prime}$. We start from the following action of $C$ on $F \dot{\cup} F^{\prime}$ :

$$
\mathbb{P} X+(c)=-\tau(\bar{c}) \mathbb{P} X, \quad-\mathbb{P} X+(c)=\tau(c) \mathbb{P} X,
$$

where $c \mapsto \bar{c}$ denotes the hyperelliptic involution. The second equality follows the idea that $\tau: C \times F \rightarrow F$ serves as a subtraction, and the first equality was rigged so that divisors linearly equivalent to the hyperellipitic class $D_{0}$ act trivially. Lemma 2.7 allows one to extend this action to the semi-group of effective divisors on $C$. Negating (2.4) then gives the extension to the entire group of divisors.

Lemma 2.7. For any $x \in F \dot{\cup} F^{\prime}$ and $c_{1}, c_{2} \in C$,

$$
\left(x+\left(c_{1}\right)\right)+\left(c_{2}\right)=\left(x+\left(c_{2}\right)\right)+\left(c_{1}\right) .
$$

Proof. Unwinding the above definition, we need to prove that for any $\mathbb{P} X \in F$ and any $c_{1}, c_{2} \in C$,

$$
\tau\left(c_{2}\right) \tau\left(\overline{c_{1}}\right) \mathbb{P} X=\tau\left(c_{1}\right) \tau\left(\overline{c_{2}}\right) \mathbb{P} X .
$$

As both sides are defined by polynomial equations, it suffices to prove this equality for generic $\mathbb{P} X$, $c_{1}, c_{2}$ over the algebraic closure; in particular, we may assume that no tangency is involved. This is proved in [Don80, Proof of Decomposition Lemma 2.6, p. 232] by looking at the intersection

$$
\operatorname{Span}\left\{\mathbb{P} X, \tau\left(\overline{c_{1}}\right) \mathbb{P} X, \tau\left(\overline{c_{2}}\right) \mathbb{P} X\right\} \cap B .
$$

ThEOREM 2.8. The above action of $\operatorname{Div}(C)$ descends to a simply transitive action of $H$ on $F \dot{\cup} F^{\prime}$. 


\section{WANG}

Remark 2.9. First, note that $+_{G}$ is defined over $k$, because $\tau$ is defined over $k$. Moreover, from the definition of the action of $\operatorname{Div}(C)$ on $F \dot{\cup} F^{\prime}$, we see that $[D]$ sends $-x^{\prime}$ to $x$ if and only if it sends $-x$ to $x^{\prime}$. Hence $+_{G}$ is commutative.

Before proving Theorem 2.8, we give some concrete examples of $+_{G}$ in certain simple cases.

EXAmple 2.10. Suppose $n=1$. Then $F$ is the variety of points in the intersection of two generic quadrics in $\mathbb{P}^{3}$ and $C$ is a genus 1 curve. Given two points $\mathbb{P} X, \mathbb{P} X^{\prime} \in F$, let $\mathbb{P} Y$ denote the line passing through them. Then $\mathbb{P} Y$ lies on a unique ruling on a unique quadric in the pencil. Such data determines a point on $C$. Identifying $C$ with $\operatorname{Pic}^{1}(C)$ naturally gives the map $+_{G}: F \times F \rightarrow \operatorname{Pic}^{1}(C)$.

EXAMPLE 2.11. Now, suppose that $n$ is general and $\mathbb{P} X, \mathbb{P} X^{\prime} \in F$ intersect in codimension 1 . Let $\mathbb{P} Y=\operatorname{Span}\left\{\mathbb{P} X, \mathbb{P} X^{\prime}\right\}$ denote their linear span. Then $\mathbb{P} Y \simeq \mathbb{P}^{n}$. Let $p$ be a point on $\mathbb{P} Y \backslash(\mathbb{P} X \cup$ $\left.\mathbb{P} X^{\prime}\right)$. There is a quadric $Q$ in $\mathcal{L}$ containing $p$. Its intersection with $\mathbb{P} Y$ contains two $\mathbb{P}^{n-1}$ and a point not on them. Hence $Q$ contains the entire $\mathbb{P} Y$. Furthermore, since the pencil is generic, the base locus contains no $\mathbb{P}^{n}$. Hence $\mathbb{P} Y$ is contained in the unique quadric $Q$ in $\mathcal{L}$ and a unique ruling on $Q$. Once again, such data determines a point on $c \in C$, and our group law says

$$
\mathbb{P} X+{ }_{G} \mathbb{P} X^{\prime}=(c) \in \operatorname{Pic}^{1}(C) .
$$

ExAmple 2.12. For any $\mathbb{P} X \in F$, since $B$ is a complete intersection,

$$
T_{\mathbb{P} X} B=T_{\mathbb{P} X} Q_{1} \cap T_{\mathbb{P} X} Q_{2}=\mathbb{P}\left(X^{\perp_{Q_{1}}} \cap X^{\perp_{Q_{2}}}\right) .
$$

As Lemma 2.13 shows, $T_{\mathbb{P} X} B$ has dimension at most $n$. If $\mathbb{P} X \in F$ is such that $T_{\mathbb{P} X} B \simeq \mathbb{P}^{n}$, then just as in the above example, $T_{\mathbb{P} X} B$ lies on a unique ruling on a unique quadric in the pencil. Such data determines a point $c \in C$, and our group law says

$$
\mathbb{P} X+{ }_{G} \mathbb{P} X=(c) \in \operatorname{Pic}^{1}(C) .
$$

As we will see in Example 2.27, for each Weierstrass point, there are $2^{2 n}$ such $\mathbb{P} X$ for which $T_{\mathbb{P} X} B$ is isomorphic to $\mathbb{P}^{n}$ and is contained in the corresponding singular quadric.

Lemma 2.13. For a generic pencil $\mathcal{L}$, we have $\operatorname{dim}\left(T_{\mathbb{P} X} B\right) \leqslant n$.

Proof. Suppose, without loss of generality, that $Q_{1}$ and $Q_{2}$ are non-degenerate. As $\operatorname{dim}(X)=n$, it follows that $\operatorname{dim}\left(X^{\perp_{Q_{i}}}\right)=n+2$ for $i=1,2$. Suppose, for a contradiction, $\operatorname{dim}\left(T_{\mathbb{P} X} B\right) \geqslant n+1$. Then $X^{\perp_{Q_{1}}}=X^{\perp_{Q_{2}}}$, and we denote this common linear $(n+2)$-plane by $H$. For any quadric $Q$ in the pencil, we have $H \subset X^{\perp_{Q}}$. Since the cone points span the entire $\mathbb{P}(U)$, there exists a cone point $\left[v_{\lambda}\right]$ of a singular quadric $Q_{\lambda} \in \mathcal{L}$ such that $v_{\lambda} \notin H$. Since $Q_{\lambda}$ descends to a quadratic form on the 2-dimensional vector space $H / X$, there exists a vector $v \in H \backslash X$ such that $Q_{\lambda}(v)=0$. Now,

$$
\operatorname{Span}\left\{X, v, v_{\lambda}\right\} \subset U
$$

is an $(n+2)$-dimensional isotropic subspace with respect to $Q_{\lambda}$. However, since $Q_{\lambda}$ is a simple quadric cone, its maximal isotropic subspace has dimension $n+1$, so we have a contradiction.

We will prove Theorem 2.8 by proving the following three propositions.

Proposition 2.14. The group $\operatorname{Div}(C)$ acts transitively on $F \dot{\cup} F^{\prime}$.

Proposition 2.15. The principal divisors act trivially on $F \dot{\cup} F^{\prime}$. Since $\left[D_{0}\right]$ acts trivially, we now have a transitive action of $H$ on $F \dot{\cup} F^{\prime}$. 


\section{PENCILS OF QUADRICS AND HYPERELLIPTIC CURVES}

Proposition 2.16. If $[D] \in H$ acts trivially, then $[D]=0$.

Without loss of generality, we assume that $k$ is algebraically closed. The following two lemmas proved in [Don80] are crucial in proving these propositions.

Lemma 2.17 ([Don80, Lemma 2.6]). Suppose that $\mathbb{P} X, \mathbb{P} X^{\prime} \in F$ intersect at codimension $r \geqslant 1$. There exists a unique effective divisor $D$ of degree $r$ such that

$$
\mathbb{P} X+D=\mathbb{P} X^{\prime} \text { if } r \text { is even, } \mathbb{P} X+D=-\mathbb{P} X^{\prime} \text { if } r \text { is odd } .
$$

In particular, there exists $\mathbb{P} X^{\prime \prime} \in F$ intersecting $\mathbb{P} X$ at codimension 1 while intersecting $\mathbb{P} X^{\prime}$ at codimension $r-1$.

Lemma 2.18 ([Don80, Lemma 3.2]). Suppose that $D$ is an effective divisor with

$$
h^{0}(D)=\operatorname{dim} H^{0}\left(\mathcal{O}_{C}(D)\right) \geqslant 2,
$$

where $H^{0}\left(\mathcal{O}_{C}(D)\right)=\{f \in k(C) \mid D+\operatorname{div}(f) \geqslant 0\}$. Then $[D]-\left[D_{0}\right]$ is also effective.

Proof of Proposition 2.14. It suffices to show that for any $\mathbb{P} X_{1}, \mathbb{P} X_{2} \in F$, there exists an element $D \in \operatorname{Div}(C)$ sending $-\mathbb{P} X_{1}$ to $\mathbb{P} X_{2}$. Let $\mathbb{P} X_{0}$ be an element of $F$ such that $T_{\mathbb{P} X_{0}} B$ is an $n$-plane. By Example 2.27, there are $2^{2 n}$ such that $\mathbb{P} X_{0}$. Then by Example 2.12, there exists a point $e \in C$ such that $-\mathbb{P} X_{0}+(e)=\mathbb{P} X_{0}$. We claim that for any $\mathbb{P} X \in F$, there exists a divisor $D \in \operatorname{Div}(C)$ sending $-\mathbb{P} X_{0}$ to $\mathbb{P} X$. Indeed, we induct on the intersection codimension $r$ of $\mathbb{P} X_{0}$ and $\mathbb{P} X$. The base case $r=0$ is when $\mathbb{P} X=\mathbb{P} X_{0}$, in which case $D=(e)$ does the job. Suppose that the claim is true for all $\mathbb{P} X^{\prime}$ intersecting $\mathbb{P} X_{0}$ at codimension at most $r-1$, and suppose that $\mathbb{P} X$ intersects $\mathbb{P} X_{0}$ at codimension $r$. By Lemma 2.17, there exists $\mathbb{P} X^{\prime} \in F$ intersecting $\mathbb{P} X$ at codimension 1 while intersecting $\mathbb{P} X_{0}$ at codimension $r-1$. By Example 2.11, there exists a point $e^{\prime} \in C$ such that $(e)$ sends $-\mathbb{P} X^{\prime}$ to $\mathbb{P} X$. Let $D^{\prime} \in \operatorname{Div}(C)$ be a divisor sending $-\mathbb{P} X_{0}$ to $\mathbb{P} X^{\prime}$. It is then easy to see that the divisor $(e)-D^{\prime}+\left(e^{\prime}\right)$ does the job.

Now, suppose that $\mathbb{P} X_{1}, \mathbb{P} X_{2} \in F$ are arbitrary. Let $D_{1}$ and $D_{2}$ denote divisors sending $-\mathbb{P} X_{0}$ to $\mathbb{P} X_{1}$ and $\mathbb{P} X_{2}$, respectively. It is easy to see that the divisor $D_{1}-(e)+D_{2}$ sends $-\mathbb{P} X_{1}$ to $\mathbb{P} X_{2}$.

Lemma 2.19. If $D \in \operatorname{Div}(C)$ fixes some $x_{0} \in F \dot{\cup} F^{\prime}$, then $D$ acts trivially.

Proof. This follows immediately from the transitivity of the action and the commutativity of $\operatorname{Div}(C)$.

Lemma 2.20. If $D$ and $E$ are effective divisors of degree at most $n$ and $D-E=\operatorname{div}(f)$ is a principal divisor, then $D-E$ acts trivially.

Proof. Applying Lemma 2.18 repeatedly to $D$, one obtains a unique effective divisor $D_{1}$ with $h^{0}\left(D_{1}\right)=1$ and such that $D$ and $E$ are in the linear system $D_{1}+\frac{1}{2}\left(\operatorname{deg}(D)-\operatorname{deg}\left(D_{1}\right)\right) D_{0}$. Since $\operatorname{deg}(D) \leqslant n$, the set $H^{0}\left(\mathcal{O}_{C}\left(\frac{1}{2}\left(\operatorname{deg}(D)-\operatorname{deg}\left(D_{1}\right)\right) D_{0}\right)\right)$ consists entirely of functions pulled back from $\mathbb{P}^{1}$. Hence $D-E$ is a linear combination of divisors of the form $(P)+(\bar{P})$, which all act trivially on $F \dot{\cup} F^{\prime}$ by construction.

In what follows, we fix a Weierstrass point $\infty$ of $C$ (defined over $k^{s}$ ).

Lemma 2.21. Suppose $D=\left(P_{1}\right)+\cdots+\left(P_{r}\right)-r(\infty) \in \operatorname{Div}(C)$ with $P_{1}, \ldots, P_{r} \neq \infty$ and $r \leqslant n$. If $D$ is linearly equivalent to $E=\left(Q_{1}\right)+\cdots+\left(Q_{r^{\prime}}\right)-r^{\prime}(\infty)$ with $Q_{1}, \ldots, Q_{r^{\prime}} \neq \infty$ and $r^{\prime} \leqslant r$, then $x+D=x+E$ for all $x \in F \dot{\cup} F^{\prime}$. 


\section{WANG}

Proof. Apply Lemma 2.20 to $\left(P_{1}\right)+\cdots+\left(P_{r}\right)$ and $\left(Q_{1}\right)+\cdots+\left(Q_{r^{\prime}}\right)+\left(r-r^{\prime}\right)(\infty)$.

Every divisor class $[D] \in J=\operatorname{Pic}^{0}(C)$ can be represented by a divisor of the form $\left(P_{1}\right)+\cdots+$ $\left(P_{r}\right)-r(\infty)$ with $P_{1}, \ldots, P_{r} \neq \infty$ and $r \leqslant n$. Lemma 2.21 says that two different representations of $[D]$ have the same action on $F \dot{\cup} F^{\prime}$. Since $\operatorname{deg}(D)$ is even, it sends $F$ to $F$. Hence we have a morphism of varieties

$$
\alpha: J \rightarrow \operatorname{Aut}(F)
$$

The image of $\alpha$ lies in a commutative subvariety of $\operatorname{Aut}(F)$. Since $J$ is complete and $\alpha([0])=\mathrm{id}$, rigidity [Mum70, II.4, pp. 40-41] implies that $\alpha$ is a group homomorphism.

Proof of Proposition 2.15. Let $\beta: \operatorname{Div}^{0}(C) \rightarrow \operatorname{Aut}(F)$ denote the action map. To show that the principal divisors act trivially, it suffices to show that $\beta$ factors through $\alpha: J \rightarrow \operatorname{Aut}(F)$. Since both are group homomorphisms, it suffices to check

$$
\beta\left((c)-\left(c^{\prime}\right)\right)=\alpha\left(\left[(c)-\left(c^{\prime}\right)\right]\right)
$$

for any $c, c^{\prime} \in C$. For any $\mathbb{P} X \in F$,

$$
\begin{aligned}
\alpha\left(\left[(c)-\left(c^{\prime}\right)\right]\right)(\mathbb{P} X) & =\mathbb{P} X+(c)+\left(\overline{c^{\prime}}\right)-2(\infty) \\
& =\mathbb{P} X+(c)-\left(c^{\prime}\right) \\
& =\beta\left((c)-\left(c^{\prime}\right)\right)(\mathbb{P} X) .
\end{aligned}
$$

We now work toward the proof of Proposition 2.16. Given two elements $x= \pm \mathbb{P} X$ and $x^{\prime}= \pm \mathbb{P} X^{\prime}$ of $F \dot{\cup} F^{\prime}$, we define their intersection codimension as the intersection codimension of $\mathbb{P} X$ and $\mathbb{P} X^{\prime}$ and write

$$
\operatorname{codim}\left(x, x^{\prime}\right)=\operatorname{codim}\left(\mathbb{P} X, \mathbb{P} X^{\prime}\right) .
$$

In this notation, Lemma 2.17 can be stated as follows.

Lemma 2.22. Suppose $x, x^{\prime} \in F$ or $x, x^{\prime} \in F^{\prime}$. Then there exists a unique effective divisor $D$ of degree $r=\operatorname{codim}\left(x, x^{\prime}\right)$ such that

$$
x+D=(-1)^{r} x^{\prime} .
$$

Lemma 2.23. Suppose that $D$ is an effective divisor of degree $r$ with $1 \leqslant r \leqslant n$. Then there exists $x \in F$ such that

$$
\operatorname{codim}(x, x+D) \equiv r \quad(\bmod 2) .
$$

There also exists $x \in F^{\prime}$ satisfying the same condition.

Proof. The case when $r=1$ follows immediately from Example 2.11. Now, suppose $r \geqslant 2$. Suppose, for a contradiction, that for all $x \in F$,

$$
\operatorname{codim}(x, x+D) \equiv r-1 \quad(\bmod 2) .
$$

Consider the closed variety

$$
\Sigma=\left\{\left(x, c_{1}, \ldots, c_{r-1}\right) \mid x \in F, c_{i} \in C, x+D=-x+\left(c_{1}\right)+\cdots+\left(c_{r-1}\right)\right\} \subset F \times \operatorname{Sym}^{r-1}(C) .
$$

Denote the two projections to $F$ and $\operatorname{Sym}^{r-1}(C)$ by $\pi_{1}$ and $\pi_{2}$, respectively. We claim that $\pi_{1}$ is surjective. Indeed, let $x$ be any element of $F$. Let $r^{\prime}$ denote the intersection codimension of $x$ and $x+D$. Then $r^{\prime} \leqslant r$. By Lemma 2.22, there exists an effective divisor $D^{\prime}$ of degree $r^{\prime}$ such that

$$
x+D=(-1)^{r-r^{\prime}}\left(x+D^{\prime}\right) .
$$




\section{PENCILS OF QUADRICS AND HYPERELLIPTIC CURVES}

Assumption (2.5) says that $r-r^{\prime}$ is odd for all $x$. Replacing $D^{\prime}$ by $D^{\prime}+\left(r-1-r^{\prime}\right)(\infty)$ shows that $\pi_{1}$ is surjective. Since $\operatorname{dim}(F)=n$ and $\operatorname{dim}\left(\operatorname{Sym}^{r-1}(C)\right)=r-1<n$, there exists a fiber of $\pi_{2}$ of positive dimension. In other words, there exists a divisor $\widetilde{D}$ of odd degree such that for infinitely many $x \in F$,

$$
x+\widetilde{D}=-x .
$$

Let $D_{1}$ be a divisor such that $2 D_{1}-(\infty)$ is linearly equivalent to $\widetilde{D}$. Since we have shown that the principal divisors act trivially, (2.6) implies that for infinitely many $x \in F$,

$$
\left(x+D_{1}\right)=-\left(x+D_{1}\right)+(\infty) .
$$

Hence for infinitely many $\mathbb{P} X \in F$,

$$
\mathbb{P} X=\tau(\infty) \mathbb{P} X .
$$

However, as we will see in Example 2.27, there are only $2^{2 n}$ such $\mathbb{P} X$, so we have a contradiction.

The statement for $F^{\prime}$ follows from the same argument, which is the main reason why we have used $x$ to denote an element of $F$ instead of the usual $\mathbb{P} X$.

Proof of Proposition 2.16. Suppose that $D=\left(P_{1}\right)+\cdots+\left(P_{r}\right)-r(\infty)$ acts trivially on $F$ with $P_{1}, \ldots, P_{r} \neq \infty$ and $r \leqslant n$ minimal.

First, suppose that $r=2 r^{\prime}$ is even. Then for all $\mathbb{P} X \in F$,

$$
\mathbb{P} X+\left(P_{1}\right)+\cdots+\left(P_{r^{\prime}}\right)=\mathbb{P} X+\left(\bar{P}_{r^{\prime}+1}\right)+\cdots+\left(\bar{P}_{r}\right) .
$$

By Lemma 2.23, there exists $\mathbb{P} X_{0} \in F$ such that

$$
\operatorname{codim}\left(\mathbb{P} X_{0}, \mathbb{P} X_{0}+\left(P_{1}\right)+\cdots+\left(P_{r^{\prime}}\right)\right)=r^{\prime \prime} \equiv r^{\prime} \quad(\bmod 2) .
$$

By the existence part of Lemma 2.17, there exist points $Q_{1}, \ldots, Q_{r^{\prime \prime}} \in C$ such that

$$
\mathbb{P} X_{0}+\left(P_{1}\right)+\cdots+\left(P_{r^{\prime}}\right)=\mathbb{P} X_{0}+\left(Q_{1}\right)+\cdots+\left(Q_{r^{\prime \prime}}\right) .
$$

The divisor $\left(Q_{1}\right)+\cdots+\left(Q_{r^{\prime \prime}}\right)+\left(P_{r^{\prime}+1}\right)+\cdots+\left(P_{r}\right)-\left(r^{\prime \prime}+r^{\prime}\right)(\infty)$ then fixes $\mathbb{P} X_{0}$ and so acts trivially on $F$ by Lemma 2.19. The minimality of $r$ forces $r^{\prime \prime}=r^{\prime}$. That is,

$$
\operatorname{codim}\left(\mathbb{P} X_{0}, \mathbb{P} X_{0}+\left(P_{1}\right)+\cdots+\left(P_{r^{\prime}}\right)\right)=r^{\prime} .
$$

By the uniqueness part of Lemma 2.17, we have

$$
\left(P_{1}\right)+\cdots+\left(P_{r^{\prime}}\right)=\left(\bar{P}_{r^{\prime}+1}\right)+\cdots+\left(\bar{P}_{r}\right)
$$

as effective divisors of degree $r^{\prime}$. Therefore, $D$ is principal.

Now, suppose that $r=2 r^{\prime}+1$ is odd. Then for all $\mathbb{P} X \in F$,

$$
\mathbb{P} X+\left(P_{1}\right)+\cdots+\left(P_{r^{\prime}+1}\right)=\mathbb{P} X+\left(\bar{P}_{r^{\prime}+2}\right)+\cdots+\left(\bar{P}_{r}\right)+(\infty) .
$$

Arguing just like in the even case, we see that the minimality of $r$ implies that for some $\mathbb{P} X_{0} \in F$,

$$
\operatorname{codim}\left(\mathbb{P} X_{0}, \mathbb{P} X_{0}+\left(P_{1}\right)+\cdots+\left(P_{r^{\prime}+1}\right)\right)=r^{\prime}+1 .
$$

Then Lemma 2.17 implies

$$
\left(P_{1}\right)+\cdots+\left(P_{r^{\prime}+1}\right)=\left(\bar{P}_{r^{\prime}+2}\right)+\cdots+\left(\bar{P}_{r}\right)+(\infty)
$$

as effective divisors of degree $r^{\prime}+1$. Therefore, $D$ is principal.

We have completed the proofs of Propositions 2.14, 2.15, and 2.16. Before moving on to state the main theorem, we describe a stronger form of Lemma 2.23 for completeness. 


\section{WANG}

Lemma 2.18 implies that if $\left(P_{1}\right)+\cdots+\left(P_{r}\right)-r(\infty)$ and $\left(Q_{1}\right)+\cdots+\left(Q_{r}\right)-r(\infty)$, with $r \leqslant n$, are two distinct divisors representing the same divisor class $[D] \in J$, then $[D]$ can also be represented by a divisor of the form $\left(R_{1}\right)+\cdots+\left(R_{r-2}\right)-(r-2)(\infty)$. Hence if $r$ is minimal among all such representations of $[D]$, there is a unique effective divisor $D^{\prime}$ of degree $r$ such that

$$
\left[D^{\prime}-r(\infty)\right]=[D] .
$$

We call $D^{\prime}$ the $\infty$-minimal form of $[D]$.

Corollary 2.24. Let $D^{\prime}$ be the $\infty$-minimal form of a non-zero divisor class $[D]$. Then there exists $x \in F$ such that

$$
\operatorname{codim}\left(x, x+D^{\prime}\right)=\operatorname{deg}\left(D^{\prime}\right) .
$$

There also exists $x \in F^{\prime}$ satisfying the same condition.

Proof. Let $r$ denote the degree of $D^{\prime}$. Lemma 2.23 allows us to pick an $x \in F$ such that

$$
\operatorname{codim}\left(x, x+D^{\prime}\right)=: r^{\prime} \equiv r \quad(\bmod 2) .
$$

By Lemma 2.22, there exists an effective divisor $D^{\prime \prime}$ of degree $r^{\prime}$ such that $x+D^{\prime}=x+D^{\prime \prime}$. Hence $D^{\prime}-D^{\prime \prime}$ fixes $x$ and by Lemma 2.19, $D^{\prime}-D^{\prime \prime}$ acts trivially on $F$. By Proposition 2.16, $D^{\prime}$ is linearly equivalent to $D^{\prime \prime}$. Since $D^{\prime}$ is the $\infty$-minimal form of $[D]$, we see that $r^{\prime}=r$.

The statement for $F^{\prime}$ follows from the same argument.

We now state our theorem in its complete form.

TheOREM 2.25. Let $G$ be the disconnected variety

$$
G=\operatorname{Pic}^{0}(C) \dot{\cup} F \dot{\cup} \operatorname{Pic}^{1}(C) \dot{\cup} F^{\prime},
$$

where $F^{\prime}$ is a copy of $F$. There is a unique commutative algebraic group structure $+_{G}$ on $G$ over $k$ such that

1. $G^{0}=\operatorname{Pic}^{0}(C)$ with component group $G / G^{0} \simeq \mathbb{Z} / 4$;

2. $F^{\prime}$ is isomorphic to $F$ as varieties via the inversion map $-1_{G}$;

3. the group law extends that on $H=\operatorname{Pic}(C) / D_{0} \simeq \operatorname{Pic}^{0}(C) \dot{\cup} \operatorname{Pic}^{1}(C)$, where $D_{0}$ is the hyperelliptic class;

4. the group law defines a simply transitive action of $H$ on $F \dot{\cup} F^{\prime}$ extending the following action of $C$ :

$$
\mathbb{P} X+(c)=-\tau(c) \mathbb{P} X, \quad-\mathbb{P} X+(c)=\tau(\bar{c}) \mathbb{P} X,
$$

with respect to which $x+{ }_{G} x^{\prime}$, for $x, x^{\prime} \in F \dot{\cup} F^{\prime}$, is the unique divisor class sending $-x$ to $x^{\prime}$.

Proof. The only thing left to check is the associativity, which amounts to the four equalities

$$
\begin{aligned}
{\left[D_{1}\right]+_{G}\left(\left[D_{2}\right]+_{G}\left[D_{3}\right]\right) } & =\left(\left[D_{1}\right]+_{G}\left[D_{2}\right]\right)+_{G}\left[D_{3}\right], \\
x+G\left(\left[D_{2}\right]+_{G}\left[D_{3}\right]\right) & =\left(x+_{G}\left[D_{2}\right]\right)+_{G}\left[D_{3}\right], \\
x+_{G}\left(x^{\prime}+_{G}\left[D_{3}\right]\right) & =\left(x+_{G} x^{\prime}\right)+_{G}\left[D_{3}\right], \\
x+G\left(x^{\prime}+_{G} x^{\prime \prime}\right) & =\left(x++_{G} x^{\prime}\right)+_{G} x^{\prime \prime}
\end{aligned}
$$

for any $\left[D_{1}\right],\left[D_{2}\right],\left[D_{3}\right] \in H$ and any $x, x^{\prime}, x^{\prime \prime} \in F \dot{\cup} F^{\prime}$.

The first one is the associativity of the group law on $H$. The second follows from the definition of the action of $H$. The third follows as both sides send $-x$ to $x^{\prime}+\left[D_{3}\right]$. For the fourth one, 


\section{PENCILS OF QUADRICS AND HYPERELLIPTIC CURVES}

denote the two sides by $x_{L}$ and $x_{R}$ and add $x^{\prime}$ to both sides. The third associativity tells us $x^{\prime}+_{G} x_{L}=\left(x^{\prime}+_{G} x\right)+_{G}\left(x^{\prime}+_{G} x^{\prime \prime}\right)$ and likewise, $x_{R}+_{G} x^{\prime}=\left(x+_{G} x^{\prime}\right)+_{G}\left(x^{\prime \prime}+_{G} x^{\prime}\right)$. The commutativity of $+_{G}$ implies that these two elements of $\operatorname{Pic}^{0}(C)$ are equal. Therefore, $x_{L}=x_{R}$ is the image of $-x^{\prime}$ under the action of $x^{\prime}+_{G} x_{L}=x^{\prime}+{ }_{G} x_{R}$.

Corollary 2.26. The class $[F] \in H^{1}(k, J)$ is 4-torsion, twice of which is $\left[\operatorname{Pic}^{1}(C)\right]$. One can lift $[F]$ to a torsor of $J[4]$ by taking

$$
F[4]:=\left\{\mathbb{P} X \in F \mid \mathbb{P} X+_{G} \mathbb{P} X+{ }_{G} \mathbb{P} X+_{G} \mathbb{P} X=0\right\} .
$$

When $\operatorname{Pic}^{1}(C)(k) \neq \emptyset$, the class $[F]$ is 2-torsion. For each $\left[D_{1}\right] \in \operatorname{Pic}^{1}(C)(k)$, we obtain a lift of $F$ to a torsor of $J[2]$ by taking

$$
F[2]_{\left[D_{1}\right]}=\left\{\mathbb{P} X \in F \mid \mathbb{P} X+_{G} \mathbb{P} X=\left[D_{1}\right]\right\} .
$$

For the remainder of this section, we consider special cases where $\left[D_{1}\right]=(c)$ comes from a point $c$ on the curve $C$.

\subsubsection{Example: Rational Weierstrass point}

ExAmple 2.27. Suppose that $C$ has a rational Weierstrass point or, equivalently, that $\mathcal{L}$ has a rational singular quadric. By moving this point to $\infty$, we assume that $Q_{1}$ is singular with cone point $\left[v_{\infty}\right]$. Let $H=v_{\infty}^{\perp Q_{2}}$ be the hyperplane in $U$ orthogonal to $v_{\infty}$ with respect to $Q_{2}$. Then $\tau(\infty)$ is induced by the linear map on $U$ that fixes $H$ and sends $v_{\infty}$ to $-v_{\infty}$. Hence

$$
F[2]_{\infty}=\{\mathbb{P} X \in F \mid \mathbb{P} X \subset B \cap \mathbb{P} H\} .
$$

Notice that when restricted to the $(2 n+1)$-dimensional vector space $H$, the two quadrics $Q_{1}$ and $Q_{2}$ span a generic pencil $\mathcal{L}_{H}$. Moreover, $Q_{1 \mid H}$ is non-degenerate. Let $T$ be the self-adjoint operator on $H$ associated to the pencil $\mathcal{L}_{H}$ as defined in (2.1). Then the right-hand side of (2.9) is precisely $W_{T}$ as defined in the odd-dimension case. Now $J[2]$ acts on $F[2]_{\infty}$ via the action of $J$ on $F$ and on $W_{T}$ via the identification $J[2] \simeq \operatorname{Stab}_{\mathrm{PO}\left(H, Q_{1 \mid H}\right)}(T)$. It is not a priori clear that these two actions are the same, for the action of $J$ on $F$ is not induced by an action on the underlying vector space. The following result says that these two actions in fact coincide.

Proposition 2.28. As $J[2]$-torsors, we have $F[2]_{\infty}=W_{T}$.

Proof. It suffices to show that for any $(P)-(\infty) \in J[2]\left(k^{s}\right)$ with $P$ a Weierstrass point, the two actions are the same. Let $\alpha$ denote the root of $f(x)$ corresponding to $P$, and set $h(x)=$ $f(x) /(x-\alpha)$. On $W_{T}\left(k^{s}\right)$, by Remark 2.6, the action of $(P)-(\infty)$ is induced by the following map on $H \otimes k^{s}$ :

$$
x \mapsto x-2 \frac{h(T)}{h(\alpha)} x .
$$

We now compute the action of $(P)-(\infty)$ on $F[2]_{\infty}\left(k^{s}\right)$. The singular quadric corresponding to $P$ is $\alpha Q_{1}-Q_{2}$. Let $w_{P} \in H \otimes k^{s}$ be an eigenvector of $T$ with eigenvalue $\alpha$. The cone point of $\alpha Q_{1}-Q_{2}$ is $\left[\left(w_{P}, 0\right)\right]$. Here, we have decomposed $U \otimes k^{s}$ as $H \oplus U_{\infty, T}$, where $U_{\infty, T}$ is the kernel of the degenerate quadric $Q_{1}$. Let $b$ denote the bilinear form associated to $Q_{1}$, and let $b_{1}$ denote the bilinear form associated to the restriction of $Q_{1}$ to $H$. From the definition of $\tau$ earlier, we see that the action of $(P)-(\infty)$ is induced by the following map on $U \otimes k^{s}$ :

$$
x \mapsto x-2 \frac{b\left(x,\left(w_{P}, 0\right)\right)}{b\left(\left(w_{P}, 0\right),\left(w_{P}, 0\right)\right)}\left(w_{P}, 0\right) .
$$




\section{WANG}

If we view each $\mathbb{P} X \in F[2]_{\infty}\left(k^{s}\right)$ as sitting inside $\mathbb{P} H$, then the action of $(P)-(\infty)$ is induced by the following map on $H \otimes k^{s}$ :

$$
x \mapsto x-2 \frac{b_{1}\left(x, w_{P}\right)}{b_{1}\left(w_{P}, w_{P}\right)} w_{P} .
$$

To prove Proposition 2.28, it remains to show that for any $x \in H \otimes k^{s}$, we have

$$
\frac{h(T)}{h(\alpha)} x=\frac{b_{1}\left(x, w_{P}\right)}{b_{1}\left(w_{P}, w_{P}\right)} w_{P} .
$$

Since both sides are killed by $T-\alpha$, and since $T$ has 1-dimensional eigenspaces, they are both scalar multiples of $w_{P}$. Now

$$
b_{1}\left(\frac{h(T)}{h(\alpha)} x, w_{P}\right)=b_{1}\left(x, \frac{h(T)}{h(\alpha)} w_{P}\right)=b_{1}\left(x, w_{P}\right)=b_{1}\left(\frac{b_{1}\left(x, w_{P}\right)}{b_{1}\left(w_{P}, w_{P}\right)} w_{P}, w_{P}\right) .
$$

Therefore, they are the same scalar multiple of $w_{P}$.

Remark 2.29. Equation (2.9) offers another viewpoint for the canonical identification of $J[2]$ with the stabilizer of a self-adjoint operator: they share a common principal homogeneous space. Fix any $k$-rational $T$, and form the corresponding pencil of quadrics. Then $J[2]$ acts on $F[2]_{\infty}$ simply transitively and $\operatorname{Stab}(T)$ acts on $W_{T}$ simply transitively. Identifying $F[2]_{\infty}$ and $W_{T}$ by Proposition 2.28, we see from the definitions that these two actions commute. Fix some $X_{0} \in F[2]_{\infty}$; one can define the map

$$
\iota: J[2] \rightarrow \operatorname{Stab}(T)
$$

by taking $\iota([D])$, for any $[D] \in J[2]$, to be the unique element of $\operatorname{Stab}(T)$ sending $X_{0}$ to $X_{0}+[D]$. The commutativity of the two actions and the commutativity of $J[2]$ show that this map is independent of the choice of $X_{0}$. Proposition 2.28 then implies that $\iota$ is given by the map we defined in Remark 2.6.

\subsubsection{Example: Rational non-Weierstrass point}

ExAmple 2.30. Suppose that $C$ has a rational non-Weierstrass point or, equivalently, that $\mathcal{L}$ has a rational quadric with discriminant 1 . By moving this point to infinity, we assume that $Q_{1}$ has discriminant 1. The two rulings of $Q_{1}$ are then defined over $k$. Let $Y_{0}$ denote one of the rulings, and let $\infty \in C(k)$ denote the point corresponding to the quadric $Q_{1}$ and the ruling $Y_{0}$. Denote by $\infty^{\prime}$ the conjugate of $\infty$ under the hyperelliptic involution. Let $T$ denote the self-adjoint operator on $U$ associated to the pencil $\mathcal{L}$ as defined in (2.1). The next result gives a geometric interpretation for the torsor $F[2]_{\infty}$ of $J[2]$.

\section{Proposition 2.31.}

$$
F[2]_{\infty}=\{\mathbb{P} X \in F \mid \mathbb{P} X=\tau(\infty) \mathbb{P} X\}=\left\{\mathbb{P} X \simeq \mathbb{P}^{n-1} \mid \operatorname{Span}\{\mathbb{P} X, \mathbb{P}(T X)\} \sim Y_{0}\right\} .
$$

The latter condition means that $\operatorname{Span}\{\mathbb{P} X, \mathbb{P}(T X)\} \simeq \mathbb{P}^{n}$ is contained in $Q_{1}$ in the ruling $Y_{0}$.

Proof. First, suppose that $\mathbb{P} X$ is an $(n-1)$-plane with $\operatorname{Span}\{\mathbb{P} X, \mathbb{P}(T X)\} \sim Y_{0}$. We show $\mathbb{P} X \in$ $F[2]_{\infty}$. Indeed, since $\operatorname{Span}\{\mathbb{P} X, \mathbb{P}(T X)\}$ is isotropic with respect to $Q_{1}$, we have $T X \subset X^{\perp_{Q_{1}}}$ and hence $\mathbb{P} X \in F$. Since $\operatorname{Span}\{\mathbb{P} X, \mathbb{P}(T X)\} \supset \mathbb{P} X$ is an $n$-plane contained in $Q_{1}$ in the ruling $Y_{0}$, we see that $\tau(\infty) \mathbb{P} X$ is the residual intersection of $\operatorname{Span}\{\mathbb{P} X, \mathbb{P}(T X)\}$ with $Q_{2}$. Finally, $\operatorname{Span}\{\mathbb{P} X, \mathbb{P}(T X)\}$ intersects $Q_{2}$ tangentially at $\mathbb{P} X$ because

$$
T X \subset T X^{\perp_{Q_{1}}} \Rightarrow T X \subset X^{\perp_{Q_{2}}} \Rightarrow T_{\mathbb{P} X} Q_{2} \supset \operatorname{Span}\{\mathbb{P} X, \mathbb{P}(T X)\} .
$$




\section{PENCILS OF QUADRICS AND HYPERELLIPTIC CURVES}

Therefore, $\mathbb{P} X \in F[2]_{\infty}$.

Conversely, suppose $\mathbb{P} X \in F[2]_{\infty}$. Let $\operatorname{Span}\{\mathbb{P} X,[p]\}$ denote the $n$-plane contained in $Q_{1}$ in the ruling $Y_{0}$, for some $p \in U \otimes k^{a}$. We show

$$
\operatorname{Span}\{\mathbb{P} X, \mathbb{P}(T X)\}=\operatorname{Span}\{\mathbb{P} X,[p]\} .
$$

Let $b_{1}$ denote the bilinear form associated to $Q_{1}$. Since $\tau(\infty) \mathbb{P} X=\mathbb{P} X$, we have the following orthogonality conditions:

$$
b_{1}(x, p)=b_{1}(x, T p)=b_{1}(p, p)=0 \quad \forall x \in X .
$$

Since $\operatorname{Span}\{\mathbb{P} X,[p]\}$ does not lie in the base locus, we have $b_{1}(p, T p)=Q_{2}(p) \neq 0$. Hence $T p$ is

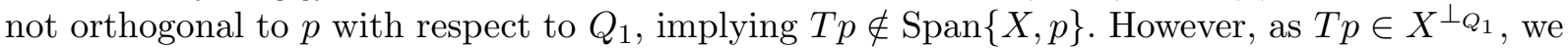
have

$$
X^{\perp_{Q_{1}}}=\operatorname{Span}\{X, p, T p\} .
$$

Since $T X \subset p^{\perp Q_{1}} \cap X^{\perp Q_{1}}$, we have $T X \subset \operatorname{Span}\{X, p\}$. Hence to obtain the desired equality (2.10), it remains to rule out the possibility $T X \subset X$. Suppose, for a contradiction, $T X \subset X$. Then $X$, being $T$-stable, contains an eigenvector of $T$. Since $T$ is regular semi-simple, its eigenvectors form an orthogonal basis with respect to $Q_{1}$. However, since $Q_{1}$ is non-degenerate, we see that none of the eigenvectors of $T$ can be isotropic. We therefore have a contradiction.

In parallel to the odd-dimension case, Proposition 2.31 suggests fixing the monic polynomial $f(x)$ of degree $2 n+2$ having no repeated roots and splitting completely over $k^{s}$, the quadratic form $Q_{1}$ of discriminant 1 , and considering the following $k$-schemes:

$$
\begin{aligned}
V_{f} & =\{T: U \rightarrow U \mid T \text { is self-adjoint with characteristic polynomial } f(x)\}, \\
W_{f} & =\left\{(T, X) \in V_{f} \times \operatorname{Gr}(n, U) \mid \operatorname{Span}\{X, T X\} \sim Y_{0}\right\} .
\end{aligned}
$$

Here $\operatorname{Span}\{X, T X\} \sim Y_{0}$ means that $\operatorname{Span}\{X, T X\}$ is a linear $(n+1)$-plane isotropic with respect to $Q_{1}$ lying in the ruling $Y_{0}$. Let $W_{T}$ denote the fiber of $W_{f}$ above $T$. Proposition 2.31 then says that $F[2]_{\infty}=W_{T}$ as $\operatorname{Gal}\left(k^{s} / k\right)$-modules. In what follows, we show that $W_{T}$ is also naturally a $J[2]$-torsor, where $J$ is the Jacobian of the hyperelliptic curve $C$ defined by the affine equation $y^{2}=f(x)$, and that $F[2]_{\infty}=W_{T}$ as $J[2]$-torsors.

The group $\mathrm{SO}\left(U, Q_{1}\right)$ preserves the rulings. Hence it acts on $W_{f}$ via

$$
g .(T, X)=\left(g T g^{-1}, g X\right) .
$$

Since the central $( \pm 1)$ acts trivially, we consider instead the action of the quotient $\operatorname{PSO}\left(U, Q_{1}\right)=$ $\mathrm{SO}\left(U, Q_{1}\right) /( \pm 1)$.

Proposition 2.32. The action of $\operatorname{PSO}\left(U, Q_{1}\right)$ on $V_{f}$ has a unique geometric orbit. For any $T \in V_{f}\left(k^{\prime}\right)$ defined over some field $k^{\prime}$ over $k$, its stabilizer scheme $\operatorname{Stab}(T)$ is isomorphic to $\left(\operatorname{Res}_{L^{\prime} / k^{\prime}} \mu_{2}\right)_{N=1} / \mu_{2} \simeq J[2]$ as group schemes over $k^{\prime}$, where $L^{\prime}=k^{\prime}[x] / f(x)$.

Proof. We prove the second statement first. Fix any $T$ in $V_{f}\left(k^{\prime}\right)$. Since $T$ is regular semi-simple, its stabilizer scheme in $\mathrm{GL}\left(U_{k^{\prime}}\right)$ is a maximal torus. It contains, and hence is equal to, the maximal torus $\operatorname{Res}_{L^{\prime} / k^{\prime}} \mathbb{G}_{m}$. For any $k^{\prime}$-algebra $K$,

$$
\begin{aligned}
\operatorname{Stab}_{O\left(U_{k^{\prime}}, Q_{1}\right)}(T)(K) & =\left\{g \in(K[T] / f(T))^{\times} \mid g^{*} g=1\right\} \\
& =\left\{g \in(K[T] / f(T))^{\times} \mid g^{2}=1\right\} .
\end{aligned}
$$




\section{WANG}

Hence

$$
\begin{aligned}
\operatorname{Stab}_{O\left(U_{k^{\prime}}, Q_{1}\right)}(T) & \simeq \operatorname{Res}_{L^{\prime} / k^{\prime}} \mu_{2}, \\
\operatorname{Stab}_{S O\left(U_{k^{\prime}}, Q_{1}\right)}(T) & \simeq\left(\operatorname{Res}_{L^{\prime} / k^{\prime}} \mu_{2}\right)_{N=1}, \\
\operatorname{Stab}_{P S O\left(U_{k^{\prime}}, Q_{1}\right)}(T) & \simeq\left(\operatorname{Res}_{L^{\prime} / k^{\prime}} \mu_{2}\right)_{N=1} / \mu_{2} .
\end{aligned}
$$

Suppose $T_{1}, T \in V_{f}\left(k^{s}\right)$. There exists $g \in G L(U)\left(k^{s}\right)$ such that $T_{1}=g T g^{-1}$. Since $T_{1}$ and $T$ are both self-adjoint, $g^{*} g$ centralizes $T$ and hence lies in $\left(k^{s}[T] / f(T)\right)^{\times}$, which is a product of $k^{s \times}$ since $f$ splits. Since the characteristic of $k$ is not 2, there exists $h \in\left(k^{s}[T] / f(T)\right)^{\times}$such that $g^{*} g=h^{2}$. Then $g h^{-1}$ is an element of $O\left(U, Q_{1}\right)\left(k^{s}\right)$ conjugating $T$ to $T_{1}$. Multiplying $h$ by $(-1,1, \ldots, 1) \in\left(k^{s}[T] / f(T)\right)^{\times}$if necessary, we may assume $g h^{-1} \in \mathrm{SO}\left(U, Q_{1}\right)\left(k^{s}\right)$. Its image in $\operatorname{PSO}\left(U, Q_{1}\right)\left(k^{s}\right)$ does the job.

For general $Q_{1}$, just like in the odd-dimension case, there might not be a self-adjoint operator defined over $k$ with the prescribed characteristic polynomial. The next result states that there is no obstruction when $Q_{1}$ is split.

Lemma 2.33. If $Q_{1}$ is split over $k$, then both $V_{f}(k)$ and $W_{f}(k)$ are non-empty. Furthermore, there exists $\left(T_{0}, X_{0}\right) \in W_{f}(k)$ with trivial stabilizer in $\operatorname{PSO}\left(U, Q_{1}\right)\left(k^{a}\right)$.

Proof. The main idea is to find a model space for the quadratic space $\left(U, Q_{1}\right)$. Consider the $(2 n+2)$-dimensional étale $k$-algebra $L=k[x] / f(x)$. Let $\beta$ denote the image of $x$ in $L$. Then $\{1, \beta$, $\left.\beta^{2}, \ldots, \beta^{2 n+1}\right\}$ forms a basis for $L$. On $L$, there is the following bilinear form:

$$
\langle\lambda, \mu\rangle=\operatorname{Tr}\left(\lambda \mu / f^{\prime}(\beta)\right)=\text { coefficient of } \beta^{2 n+1} \text { in } \lambda \mu .
$$

This form defines a split quadratic form $Q_{0}$ since $Y=\operatorname{Span}_{k}\left\{1, \beta, \ldots, \beta^{n}\right\}$ is a rational isotropic subspace of dimension $n+1$. Hence there exists an isometry from $\left(L, Q_{0}\right)$ to $\left(U, Q_{1}\right)$ defined over $k$. Under this isometry, the multiplication-by- $\beta$ operator maps to a self-adjoint operator, denoted by $T_{0}$, on $L$, while the $n$-plane $X=\operatorname{Span}_{k}\left\{1, \beta, \ldots, \beta^{n-1}\right\}$ maps to an $n$-plane, denoted by $X_{0}$, in $L$. Then $\left(T_{0}, X_{0}\right) \in W_{f}(k)$. Since $(\cdot \beta, X)$ has trivial stabilizer in $\operatorname{PSO}\left(L, Q_{0}\right)\left(k^{a}\right)$, its image $\left(T_{0}, X_{0}\right)$ has trivial stabilizer in $\operatorname{PSO}\left(U, Q_{1}\right)\left(k^{a}\right)$.

Theorem 2.34. Suppose that $k$ is separably closed of characteristic not 2. Then $\operatorname{PSO}\left(U, Q_{1}\right)(k)$ acts simply transitively on $W_{f}(k)$.

Proof. Proposition 2.32 shows that it suffices to prove that for the $T_{0} \in V_{f}(k)$ obtained in Lemma 2.33, the group $\operatorname{Stab}\left(T_{0}\right)(k)$ acts simply transitively on $W_{T_{0}}(k)$. Since $\left(T_{0}, X_{0}\right)$ has trivial stabilizer, it suffices to show that $W_{T_{0}}(k)$ and $\operatorname{Stab}\left(T_{0}\right)(k)$ have the same size. As a consequence of Proposition 2.31, for any $k$, the set $W_{T}\left(k^{a}\right) \simeq F[2]_{\infty}\left(k^{a}\right) \simeq J[2]\left(k^{a}\right)$ has $2^{2 n}$ elements for any $T$. Hence we are done, because

$$
2^{2 n}=\left|\left(\operatorname{Res}_{L / k} \mu_{2} / \mu_{2}\right)_{N=1}(k)\right|=\left|\operatorname{Stab}\left(T_{0}\right)(k)\right| \leqslant\left|W_{T_{0}}(k)\right| \leqslant\left|W_{T_{0}}\left(k^{a}\right)\right|=2^{2 n} .
$$

Corollary 2.35. Suppose that $k$ is any field of characteristic not 2 and $W_{f}(k)$ is non-empty. Then $\operatorname{PSO}\left(U, Q_{1}\right)\left(k^{\prime}\right)$ acts simply transitively on $W_{f}\left(k^{\prime}\right)$ for any field $k^{\prime}$ over $k$.

Proof. It suffices to prove transitivity. Suppose that $\left(T_{1}, X_{1}\right)$ and $\left(T_{2}, X_{2}\right)$ are in $W_{f}\left(k^{\prime}\right)$. Let $g \in$ $\operatorname{PSO}\left(U, Q_{1}\right)\left(k^{\prime s}\right)$ be the unique element sending $\left(T_{1}, X_{1}\right)$ to $\left(T_{2}, X_{2}\right)$. Then for any $\sigma \in \operatorname{Gal}\left(k^{\prime s} / k^{\prime}\right)$, the element ${ }^{\sigma} g$ also sends $\left(T_{1}, X_{1}\right)$ to $\left(T_{2}, X_{2}\right)$. Hence $g={ }^{\sigma} g$ and so $g \in \operatorname{PSO}\left(U, Q_{1}\right)\left(k^{\prime}\right)$ by separable descent. 


\section{PENCILS OF QUADRICS AND HYPERELLIPTIC CURVES}

Remark 2.36. One can write down an explicit formula for the identification

$$
\operatorname{Stab}(T) \simeq\left(\operatorname{Res}_{L / k} \mu_{2}\right)_{N=1} / \mu_{2} \simeq J[2] .
$$

The method is the same as the odd case in Remark 2.6. Denote the roots of $f(x)$ over $k^{s}$ by $\alpha_{1}, \ldots, \alpha_{2 n+2}$, and for each $i$, define $h_{i}(x)=f(x) /\left(x-\alpha_{i}\right)$. Then on the level of $k^{s}$-points, (2.11) is given by sending

$$
\sum n_{i}\left(\alpha_{i}\right)-\frac{\sum n_{i}}{2}\left((\infty)+\left(\infty^{\prime}\right)\right), \quad \sum n_{i} \text { even },
$$

to the image in $\mathrm{PSO}_{2 n+2}\left(k^{s}\right)$ of

$$
\prod\left(1-2 \frac{h_{i}(T)}{h_{i}\left(\alpha_{i}\right)}\right)^{n_{i}}=1-2 \sum n_{i} \frac{h_{i}(T)}{h_{i}\left(\alpha_{i}\right)} .
$$

Just as in Proposition 2.28, the map $1-2 h_{i}(T) / h_{i}\left(\alpha_{i}\right)$ is a reflection and so has determinant -1 . The assumption that $\sum n_{i}$ is even ensures that the product in (2.12) lies in SO.

Just as in the odd case, $J[2]$ acts on $F[2]_{\infty}$ via the action of $J$ and on $W_{T}$ via the identification $J[2] \simeq \operatorname{Stab}_{\mathrm{PSO}\left(U, Q_{1}\right)}(T)$. The following result says that these two actions coincide.

Proposition 2.37. As $J[2]$-torsors, $F[2]_{\infty}=W_{T}$.

Proof. It suffices to show that for any $\left(P_{1}\right)-\left(P_{2}\right) \in J[2]$, where $P_{1}$ and $P_{2}$ are any two Weierstrass points, the two actions are the same. Let $\alpha_{i}$ denote the root of $f(x)$ corresponding to $P_{i}$, and set $h_{i}(x)=f(x) /\left(x-\alpha_{i}\right)$ for $i=1,2$. On $W_{T}\left(k^{a}\right)$, by Remark 2.36, the action of $\left(P_{1}\right)-\left(P_{2}\right)$ is induced by the following map on $U \otimes k^{s}$ :

$$
x \mapsto x-2 \frac{h_{1}(T)}{h_{1}\left(\alpha_{1}\right)} x-2 \frac{h_{2}(T)}{h_{2}\left(\alpha_{2}\right)} x .
$$

For $i=1,2$, let $w_{i} \in U \otimes k^{s}$ be an eigenvector of $T$ with eigenvalue $\alpha_{i}$. The cone point of the singular quadric corresponding to $P_{i}$ is then $\left[w_{i}\right]$. Let $b_{1}$ denote the bilinear form associated to $Q_{1}$. Then on $F\left(k^{s}\right)$, the action of $\tau\left(P_{i}\right)$ is induced by the following map on $U \otimes k^{a}$ :

$$
\operatorname{refl}_{P_{i}}: x \mapsto x-2 \frac{b_{1}\left(x, w_{i}\right)}{b_{1}\left(w_{i}, w_{i}\right)} w_{i}
$$

Composing two such reflections, we see that the action of $\tau\left(P_{1}\right) \tau\left(P_{2}\right)$ is induced by the following map on $U \otimes k^{a}$ :

$$
x \mapsto x-2 \frac{b_{1}\left(x, w_{1}\right)}{b_{1}\left(w_{1}, w_{1}\right)} w_{1}-2 \frac{b_{1}\left(x, w_{2}\right)}{b_{1}\left(w_{2}, w_{2}\right)} w_{2}+\frac{4 b_{1}\left(x, w_{1}\right) b_{1}\left(w_{1}, w_{2}\right)}{b_{1}\left(w_{1}, w_{1}\right) b_{1}\left(w_{2}, w_{2}\right)} w_{2} .
$$

Since self-adjoint operators have pairwise orthogonal eigenspaces, the last term is 0 . Also as in the proof of Proposition 2.28, for $i=1,2$,

$$
\frac{h_{i}(T)}{h_{i}\left(\alpha_{i}\right)} x=\frac{b_{1}\left(x, w_{i}\right)}{b_{1}\left(w_{i}, w_{i}\right)} w_{i}
$$

Therefore, the two actions are equal.

Remark 2.38. In parallel to the odd case, the equality $F[2]_{\infty}=W_{T}$ as $\operatorname{Gal}\left(k^{s} / k\right)$-sets provides a different viewpoint on the identification of $J[2]$ with $\operatorname{Stab}(T)$, as they share a common principal homogeneous space. Proposition 2.37 implies that this new identification coincides with the formula given by Remark 2.36. 


\section{WANG}

\section{Regular pencils}

For the rest of the paper, we focus on regular pencils. Let $\Omega_{1}$ denote the discriminant hypersurface in $\mathbb{P}\left(H^{0}\left(\mathcal{O}_{\mathbb{P}^{N-1}}(2)\right)\right)$ parameterizing singular quadrics, and let $\Omega_{2}$ denote the subvariety parameterizing quadrics with higher degeneracy degree. Recall that a pencil $\mathcal{L}$ is generic if and only if $\mathcal{L}$ is a generic line intersecting $\Omega_{1}$ transversely at $N$ points and missing $\Omega_{2}$. A pencil is regular if it misses $\Omega_{2}$ but is allowed to intersect $\Omega_{1}$ tangentially.

In the $\left(Q_{1}, T\right)$-setup, where $Q_{1}$ is non-degenerate and $T$ is self-adjoint with respect to $Q_{1}$, regularity of the pencil is equivalent to regularity of $T$. An operator $T$ is regular if and only if its characteristic polynomial coincides with its minimal polynomial if and only if all of its eigenspaces are 1-dimensional. Let $f(x)=\operatorname{det}(x I-T)$ denote the minimal polynomial of $T$; as before, we assume that $f(x)$ splits completely over $k^{s}$.

The following reduction step is crucial to the study of the variety of maximal linear spaces contained in the base locus over $k^{s}$. Suppose, temporarily, that $k$ is separably closed. Let $U$ denote the underlying $N$-dimensional $k$-vector space. Let $v \in U$ denote an eigenvector of $T$ whose eigenvalue $\alpha$ has multiplicity at least 2 , and write $\langle v\rangle$ for the line spanned by $v$. Since $v$ is an eigenvector and $T$ is self-adjoint, $T$ descends to a linear operator $\bar{T}$ on $\bar{U}=v^{\perp} /\langle v\rangle$, where $\perp$ is taken with respect to $Q_{1}$. The quadratic form $Q_{1}$ descends to a non-degenerate quadratic form $\bar{Q}_{1}$ on $\bar{U}$ with respect to which $\bar{T}$ is regular self-adjoint with minimal polynomial $f(x) /(x-\alpha)^{2}$. Suppose $N=2 n+1$ or $2 n+2$. Let $X$ be an $n$-plane in $U$ such that $X \subset X^{\perp}$ and $T X \subset X^{\perp}$. Define $\bar{X}$ to be the image of $X \cap v^{\perp}$ in $v^{\perp} /\langle v\rangle$. Then $\bar{X} \subset \bar{X}^{\perp}$ and $\overline{T X} \subset \bar{X}^{\perp}$. As we will see in what follows, either $v \in X$ or $X \nsubseteq v^{\perp}$, and so $\operatorname{dim} \bar{X}=n-1$. The strategy will be to apply this reduction repeatedly until $T$ becomes regular semi-simple, in which case one can use the result in the previous section on generic pencils.

Factor $f(x)$ as $f(x)=\prod_{i=1}^{r+1}\left(x-\alpha_{i}\right)^{m_{i}}$ over $k^{s}$. Let $U_{i, T}$ denote the generalized eigenspace over $k^{s}$ of $T$ with eigenvalue $\alpha_{i}$, and let $v_{i}$ be an eigenvector of $T$ with eigenvalue $\alpha_{i}$. Regularity is equivalent to saying that each $v_{i}$ is unique up to scalars. The singular locus of $B$ consists precisely of all the $\left[v_{i}\right]$ with $m_{i} \geqslant 2$. For any linear $n$-plane $X$ such that $X \subset X^{\perp}$ and $T X \subset X^{\perp}$ and for each $i=1, \ldots, r+1$, we define $\operatorname{dim}_{i, T}(X)$ to be the dimension of the maximal $T$-stable subspace of $\left(X \otimes k^{s}\right) \cap U_{i, T}$. The generalized eigenspaces of $T$ are pairwise orthogonal and so $Q_{1}$ restricts to a non-degenerate quadratic form on $U_{i, T}$. Since each $U_{i, T}$ is $m_{i}$-dimensional, we have

$$
\operatorname{dim}_{i, T}(X) \leqslant m_{i} / 2 .
$$

For any sequence of integers $d_{1}, \ldots, d_{r+1}$ such that $0 \leqslant d_{i} \leqslant m_{i} / 2$, we define for any field $k^{\prime}$ containing $k$,

$$
L_{\left\{d_{1}, \ldots, d_{r+1}\right\}}^{f, T}\left(k^{\prime}\right)=\left\{X \simeq\left(k^{\prime}\right)^{n} \mid X \subset X^{\perp}, T X \subset X^{\perp}, \operatorname{dim}_{i, T}(X)=d_{i}\right\} .
$$

The superscript $f$ is unnecessary, but it serves in making the reduction step clearer. Observe that $L_{\{0,0, \ldots, 0\}}^{f, T}\left(k^{\prime}\right)$ is the set of $k^{\prime}$-points of the variety of projective $(n-1)$-planes contained in the smooth locus of $B$.

\subsection{Odd dimension}

Suppose that $N=2 n+1$ is odd. For ease of notation, we write $Q$ for the non-degenerate quadratic form $Q_{1}$. By multiplying $Q$ by a constant, we also assume that $Q$ has discriminant 1 . Fix the minimal polynomial $f(x)$ of degree $2 n+1$. Let $C$ be the hyperelliptic curve defined by the affine 


\section{PENCILS OF QUADRICS AND HYPERELLIPTIC CURVES}

equation $y^{2}=f(x)$. We define the $k$-scheme

$$
V_{f}=\{T: U \rightarrow U \mid T \text { is self-adjoint and regular with characteristic polynomial } f(x)\} .
$$

Note that regularity is equivalent to requiring that there are no linear relations among $1, T, \ldots$, $T^{2 n}$. The same argument as in the proof of Lemma 2.33 gives that $V_{f}\left(k^{\prime}\right)$ is non-empty for any field $k^{\prime}$ over which $Q$ is split.

For every field $k^{\prime}$ containing $k$ and every $T \in V_{f}\left(k^{\prime}\right)$, let $W_{T}\left(k^{\prime}\right)$ denote the set of (linear) $n$-dimensional $k^{\prime}$-subspaces $X$ of $U \otimes k^{\prime}$ such that $X \subset X^{\perp}$ and $T X \subset X^{\perp}$. As before, we define

$$
W_{f}\left(k^{\prime}\right)=\left\{(T, X) \mid T \in V_{f}\left(k^{\prime}\right), X \in W_{T}\left(k^{\prime}\right)\right\} .
$$

There is a Galois-equivariant action of $\mathrm{PO}(U, Q)=O(U, Q) /( \pm 1)$ on $W_{f}$ :

$$
g \cdot(T, X)=\left(g T g^{-1}, g X\right) \text {. }
$$

For any sequence of integers $d_{1}, \ldots, d_{r+1}$ such that $0 \leqslant d_{i} \leqslant m_{i} / 2$, we define

$$
W_{\left\{d_{1}, \ldots, d_{r+1}\right\}}^{f}\left(k^{\prime}\right)=\left\{(T, X) \mid T \in V_{f}\left(k^{\prime}\right), X \in L_{\left\{d_{1}, \ldots, d_{r+1}\right\}}^{f, T}\left(k^{\prime}\right)\right\} .
$$

The rest of the section is dedicated to proving the follow two theorems.

Theorem 3.1. Let a denote the number of $d_{i}$ equal to $m_{i} / 2$. Then

$$
\left|L_{\left\{d_{1}, \ldots, d_{r+1}\right\}}^{f, T}\left(k^{s}\right)\right|=2^{r} / 2^{a} .
$$

The action of $\mathrm{PO}(U, Q)$ preserves the decomposition of $U \otimes k^{s}$ into generalized eigenspaces, in the sense that

$$
U_{i, g T g^{-1}}=g U_{i, T} \quad \forall T \in V_{f}\left(k^{s}\right), \forall g \in \mathrm{PO}(U, Q)\left(k^{s}\right), \forall i=1, \ldots, r+1 .
$$

Hence one obtains a Galois-equivariant action of $\mathrm{PO}(U, Q)\left(k^{s}\right)$ on $W_{\left\{d_{1}, \ldots, d_{r+1}\right\}}^{f}\left(k^{s}\right)$.

THEOREM 3.2. The group $\mathrm{PO}(U, Q)\left(k^{s}\right)$ acts on $W_{\left\{d_{1}, \ldots, d_{r+1}\right\}}^{f}\left(k^{s}\right)$ simply transitively if $a=0$ and transitively if $a>0$.

Corollary 3.3. For any field $k^{\prime}$ over $k$ such that $W_{\{0, \ldots, 0\}}^{f}\left(k^{\prime}\right)$ is non-empty, $\operatorname{PO}(U, Q)\left(k^{\prime}\right)$ acts simply transitively on $W_{\{0, \ldots, 0\}}^{f}\left(k^{\prime}\right)$.

Proof. Given Theorem 3.2, the corollary follows by the same descent argument as in the proof of Corollary 2.35.

We begin by studying the conjugation action of $\mathrm{PO}(U, Q)$ on $V_{f}$ and computing the stabilizers. This allows us to reduce Theorem 3.2 to two counting results: Theorems 3.1 and 3.6.

Proposition 3.4. The action of $\mathrm{PO}(U, Q)$ on $V_{f}$ has a unique geometric orbit. For any $T \in V_{f}\left(k^{\prime}\right)$ defined over some field $k^{\prime}$ over $k$, its stabilizer scheme $\operatorname{Stab}(T)$ is isomorphic to $\operatorname{Res}_{L^{\prime} / k^{\prime}} \mu_{2} / \mu_{2} \simeq$ $J[2]$ as group schemes over $k^{\prime}$, where $L^{\prime}=k^{\prime}[x] / f(x)$. In particular, $\operatorname{Stab}_{\mathrm{PO}(U, Q)}(T)\left(k^{s}\right)$ is an elementary abelian 2-group of order $2^{r}$, where $r+1$ is the number of distinct roots $f(x)$ over $k^{s}$.

Proof. The first statement follows in the same way as in the proof of Proposition 2.32, except that now, $k^{s}[x] / f(x)$ is a product of algebras of the form $k^{s}[x] /\left(x^{m_{i}}\right)$. Every unit in $k^{s}[x] /\left(x^{m_{i}}\right)$ is a square as $\operatorname{char}(k) \neq 2$.

The second statement follows from the structure theory of finitely generated modules over principal ideal domains. One can view $U \otimes k^{\prime}$ as a module over $k^{\prime}[x]$, with $x$ acting via the 


\section{WANG}

operator $T$. The elements in $\operatorname{GL}(U)(k)$ commuting with $T$ are precisely the automorphisms of $U$ as $k^{\prime}[x]$-modules. Since $T$ is regular, the structure theory of finitely generated modules over principal ideal domains says that $U \otimes k^{\prime}$ is isomorphic to $k^{\prime}[x] / f(x)$ as a $k^{\prime}[x]$-module. As a module of $k^{\prime}[x]$ generated by the element 1 , the automorphisms of $U$ are precisely multiplication by elements in $\left(k^{\prime}[x] / f(x)\right)^{\times}$. Then as in the proof of Proposition 2.32, we write ${ }^{*}$ for the operation of taking adjoint with respect to $Q$, and we have

$$
\begin{aligned}
\operatorname{Stab}_{O(U, Q)}(T)\left(k^{\prime}\right) & =\left\{g(T) \mid g \in k^{\prime}[x], g(T)^{*} g(T)=1\right\} \\
& =\mu_{2}\left(k^{\prime}[T]^{\times}\right), \\
\operatorname{Stab}_{\mathrm{PO}(U, Q)}(T)\left(k^{\prime}\right) & =\mu_{2}\left(k^{\prime}[T]^{\times}\right) /( \pm 1) .
\end{aligned}
$$

For the last statement, from the factorization of $f(x)$, we know

$$
k^{s}[x] / f(x) \simeq \prod_{i=1}^{r+1} k^{s}[x] /\left(x-\alpha_{i}\right)^{m_{i}} .
$$

Hence $\operatorname{Stab}_{O(U, Q)}(T)\left(k^{s}\right) \simeq(\mathbb{Z} / 2 \mathbb{Z})^{r+1}$ is an elementary abelian 2-group of order $2^{r+1}$. Modding out the diagonally embedded $\mathbb{Z} / 2 \mathbb{Z}$ gives $\operatorname{Stab}_{\mathrm{PO}(U, Q)}(T)\left(k^{s}\right)$.

Remark 3.5. Just as in Remark 2.6, we can give a more explicit description for the stabilizer in terms of polynomials in $T$. For each $i=1, \ldots, r+1$, define $h_{i}^{T}(x)=f(x) /\left(x-\alpha_{i}\right)^{m_{i}}$. Then

$$
\begin{aligned}
\mu_{2}\left(k^{s}[T]^{\times}\right) & =\left\{\prod_{i \in I}\left(1-2 \frac{h_{i}^{T}(T)}{h_{i}^{T}\left(\alpha_{i}\right)}\right)\right\}_{I \subset\{1, \ldots, r+1\}} \\
& =\left\{1-2 \sum_{i \in I} \frac{h_{i}^{T}(T)}{h_{i}^{T}\left(\alpha_{i}\right)}\right\}_{I \subset\{1, \ldots, r+1\}} .
\end{aligned}
$$

Note that for any $I \subset\{1, \ldots, r+1\}$ and any $j \notin I$, since $\left(x-\alpha_{j}\right)^{m_{j}}$ divides $h_{i}^{T}(x)$ in $k^{s}[x]$ and $\left(T-\alpha_{j}\right)^{m_{j}}$ kills the generalized eigenspace $U_{j, T}$, we see that

$$
1-2 \sum_{i \in I} \frac{h_{i}^{T}(T)}{h_{i}^{T}\left(\alpha_{i}\right)}
$$

acts trivially on $U_{j, T}$.

For any $T \in V_{f}\left(k^{s}\right)$, its stabilizer $J_{T}$ in $\mathrm{PO}(U, Q)\left(k^{s}\right)$ acts on $L_{\left\{d_{1}, \ldots, d_{r+1}\right\}}^{f, T}\left(k^{s}\right)$. We rephrase Theorem 3.2 as follows.

Theorem 3.6. Let a denote the number of $d_{i}$ equal to $m_{i} / 2$. Then for any $X \in L_{\left\{d_{1}, \ldots, d_{r+1}\right\}}^{f, T}\left(k^{s}\right)$,

$$
\left|\operatorname{Stab}_{J_{T}}(X)\right|=2^{a} .
$$

Theorem 3.2 follows from Theorems 3.1 and 3.6 because the size of each orbit is

$$
\left|J_{T}\right| /\left|\operatorname{Stab}_{J_{T}}(X)\right|=2^{r} / 2^{a}=\left|L_{\left\{d_{1}, \ldots, d_{r+1}\right\}}^{f, T}\left(k^{s}\right)\right| .
$$

We will prove Theorems 3.1 and 3.6 via a series of reductions to the case of generic pencils.

Reduction on $d_{1}, \ldots, d_{r+1}$. Let $X$ be any element of $L_{\left\{d_{1}, \ldots, d_{r+1}\right\}}^{f, T}\left(k^{s}\right)$ with $d_{i} \geqslant 1$ for some $i$. Let $v_{i}$ denote an eigenvector of $T$ corresponding to $\alpha_{i}$. Since $T$ is regular, $v_{i}$ is unique up to 


\section{PENCILS OF QUADRICS AND HYPERELLIPTIC CURVES}

scaling. The assumption $d_{i} \geqslant 1$ then implies $v_{i} \in X$. Let $b$ denote the bilinear form associated to $Q$. Note that we have $v_{i} \in v_{i}^{\perp}$ since there exists some $v_{i}^{\prime}$ such that $\left(T-\alpha_{i}\right) v_{i}^{\prime}=v_{i}$, and hence

$$
b\left(v_{i}, v_{i}\right)=b\left(v_{i},\left(T-\alpha_{i}\right) v_{i}^{\prime}\right)=b\left(\left(T-\alpha_{i}\right) v_{i}, v_{i}^{\prime}\right)=0 .
$$

For any $w \in v_{i}^{\perp}$,

$$
b\left(v_{i}, T w\right)=b\left(T v_{i}, w\right)=b\left(\alpha_{i} v_{i}, w\right)=0 .
$$

Hence $T$ descends to a linear map

$$
\bar{T}_{i}: v_{i}^{\perp} /\left\langle v_{i}\right\rangle \rightarrow v_{i}^{\perp} /\left\langle v_{i}\right\rangle=: \bar{U}_{i} .
$$

The quadratic form $Q$ descends to a non-degenerate quadratic form $\bar{Q}_{i}$ with respect to which $\bar{T}_{i}$ is regular self-adjoint with characteristic polynomial $f(x) /\left(x-\alpha_{i}\right)^{2}$. Note that this reduction can be described projectively as intersecting the quadric defined by $Q$ with the tangent plane to $v_{i}$, then projecting away from $v_{i}$.

Since $v_{i} \in X$ and $X$ is isotropic, we see $X \subset v_{i}^{\perp}$. Let $\bar{X}_{i}$ denote the image of $X$ in $\bar{U}_{i}$. It is immediate from the definition that $\bar{X}_{i}$ is $(n-1)$-dimensional, satisfying

$$
\bar{X}_{i} \subset \bar{X}_{i}^{\perp} \bar{Q}_{i}, \quad \bar{T}_{i} \bar{X}_{i} \subset \bar{X}_{i}^{\perp} \bar{Q}_{i},
$$

and the dimensions of the maximal $\bar{T}_{i}$-stable subspaces in its intersection with the generalized eigenspaces are $d_{1}, \ldots, d_{i}-1, \ldots, d_{r+1}$. We denote this reduction step by

$$
\delta_{i}: L_{\left\{d_{1}, \ldots, d_{r+1}\right\}}^{f, T}\left(k^{s}\right) \stackrel{\sim}{\longrightarrow} L_{\left\{d_{1}, \ldots, d_{i}-1, \ldots, d_{r+1}\right\}}^{f /\left(x-\alpha_{i}\right)^{2}, \bar{T}_{i}}\left(k^{s}\right) .
$$

Observe that $\delta_{i}$ is bijective with its inverse given by taking the pre-image of the projection $\operatorname{map} v_{i}^{\perp} \rightarrow v_{i}^{\perp} /\left\langle v_{i}\right\rangle$.

How are the stabilizers affected by this reduction? If $h(x)$ is any polynomial in $k^{s}[x]$, then $\delta(h(T) X)=h\left(\bar{T}_{i}\right) \bar{X}_{i}$. Since $\delta$ is bijective, we conclude that $h(T)$ stabilizes $X$ if and only if $h\left(\bar{T}_{i}\right)$ stabilizes $\bar{X}_{i}$. Note that if $m_{i} \geqslant 3$, then

$$
h_{i}^{T}(x)=\frac{f(x)}{\left(x-\alpha_{i}\right)^{m_{i}}}=\frac{f(x) /\left(x-\alpha_{i}\right)^{2}}{\left(x-\alpha_{i}\right)^{m_{i}-2}}=h_{i}^{\bar{T}_{i}}(x) .
$$

Hence according to the explicit description given in Remark 3.5,

$$
h(T) \in J_{T} \Longleftrightarrow h\left(\bar{T}_{i}\right) \in J_{\bar{T}_{i}} ; \quad \text { hence }\left|\operatorname{Stab}_{J_{T}}(X)\right|=\left|\operatorname{Stab}_{\bar{T}_{i}}\left(\bar{X}_{i}\right)\right| \text {. }
$$

When $m_{i}=2$ and $d_{i}=1$, we no longer have $\alpha_{i}$ as an eigenvalue for $\bar{T}_{i}$. In this case,

$$
J_{T}=\left\langle h(T), 1-2 h_{i}(T) / h_{i}\left(\alpha_{i}\right) \mid h\left(\bar{T}_{i}\right) \in J_{\bar{T}_{i}}\right\rangle .
$$

Now $U_{i, T}=\operatorname{Span}\left\{v_{i}, v_{i}^{\prime}\right\}$ with $b\left(v_{i}, v_{i}^{\prime}\right) \neq 0$. Since $v_{i} \in X$ and $X$ is isotropic, we have

$$
X=\operatorname{Span}\left\{v_{i}, X \cap \operatorname{Span}\left\{U_{j, T}\right\}_{j \neq i}\right\} .
$$

Now $1-2 h_{i}(T) / h_{i}\left(\alpha_{i}\right)$ sends $v_{i}$ to $-v_{i}$ and fixes every element in $\operatorname{Span}\left\{U_{j, T}\right\}_{j \neq i}$. Hence it stabilizes $X$ and so

$$
\left|\operatorname{Stab}_{J_{T}}(X)\right|=2\left|\operatorname{Stab}_{\bar{T}_{i}}\left(\bar{X}_{i}\right)\right| .
$$

Note that this case is precisely when $a$ decreases by 1 in this reduction step.

We summarize this reduction step in the following proposition.

Proposition 3.7. Suppose $d_{i} \geqslant 1$; then there is a bijection

$$
\delta_{i}: L_{\left\{d_{1}, \ldots, d_{r+1}\right\}}^{f, T}\left(k^{s}\right) \stackrel{\sim}{\longrightarrow} L_{\left\{d_{1}, \ldots, d_{i}-1, \ldots, d_{r+1}\right\}}^{f /\left(x-\alpha_{i}\right)^{2}, \bar{T}_{i}}\left(k^{s}\right) .
$$




\section{WANG}

When $m_{i} \geqslant 3$, the map $\delta_{i}$ does not change the sizes of the stabilizers. When $m_{i}=2$, the map $\delta_{i}$ lowers the sizes of the stabilizers by a factor of 2 .

Reduction on $f$. By the above reduction step, it remains to study $L_{\{0,0, \ldots, 0\}}^{f, T}\left(k^{s}\right)$. We do so by applying similar reduction steps to remove the repeated factors from $f(x)$. Most of the proofs in this subsection are purely linear algebra. We recommend the interested readers to prove these linear algebra statements themselves.

Suppose that $\alpha$ is a root of $f$ of multiplicity $m \geqslant 2$. Let $X \in L_{\{0,0, \ldots, 0\}}^{f, T}\left(k^{s}\right)$ be arbitrary. Let $v$ denote an eigenvector of $T$ with eigenvalue $\alpha$. As before, we can consider the descent to $\bar{U}=$ $v^{\perp} /\langle v\rangle$. As in the above reduction step, $Q$ descends to a non-degenerate quadratic form $\bar{Q}$ on $\bar{U}$, and $T$ descends to a regular self-adjoint operator $\bar{T}$ with characteristic polynomial $f(x) /(x-\alpha)^{2}$.

Observe that we have $v \notin X$ since $X$ contains no $T$-stable subspace. Hence the map $U \rightarrow$ $U /\langle v\rangle$ is bijective when restricted to $X$. Consequently, $X \nsubseteq v^{\perp}$, for otherwise the $(2 n-1)$ dimensional vector space $v^{\perp} /\langle v\rangle$ would contain an $n$-dimensional isotropic subspace, which is impossible. Now, $X \cap v^{\perp}$ has dimension $n-1$, and we denote its bijective image in $v^{\perp} /\langle v\rangle$ by $\bar{X}$.

Proposition 3.8. The above map sending $X$ to $\bar{X}$ defines a surjection

$$
L_{\{0,0, \ldots, 0\}}^{f, T}\left(k^{s}\right) \longrightarrow L_{\{0,0, \ldots, 0\}}^{f /(x-\alpha)^{2}, \bar{T}}\left(k^{s}\right) .
$$

This map is bijective if $m \geqslant 3$ and is two-to-one if $m=2$. In both cases,

$$
\left|\operatorname{Stab}_{J_{T}}(X)\right|=\left|\operatorname{Stab}_{J_{\bar{T}}}(\bar{X})\right| \quad \text { for any } X \in L_{\{0, \ldots, 0\}}^{f, T}\left(k^{s}\right) \text {. }
$$

Proof. It is clear that $\bar{X}$ satisfies $\bar{X} \subset \bar{X}^{\perp}$ and $\overline{T X} \subset \bar{X}^{\perp}$. We claim that $\bar{X}$ contains no $\bar{T}$-stable subspace. Since $X$ contains no $T$-stable subspace, the only $\bar{T}$-stable subspace $\bar{X}$ could possibly contain is the line spanned by $v^{\prime}+\langle v\rangle$, where $v^{\prime}$ is an element of $U$ with $(T-\alpha) v^{\prime}=v$. Suppose, for a contradiction, that $X$ contains $v^{\prime}+c v$ for some $c \in k^{s}$. Then $v=(T-\alpha)\left(v^{\prime}+c v\right) \in X^{\perp}$, contradicting $X \nsubseteq v^{\perp}$. Hence $\bar{X} \in L_{\{0,0, \ldots, 0\}}^{f /(x-\alpha)^{2}, \bar{T}}\left(k^{s}\right)$.

Next, we prove surjectivity, compute the sizes of the fibers, and compare the sizes of the stabilizers. Suppose $\bar{X} \in L_{\{0,0, \ldots, 0\}}^{f /(x-\alpha)^{2}, \bar{T}}\left(k^{s}\right)$. Let $b_{\alpha}$ denote the bilinear form on $U$ defined by

$$
b_{\alpha}\left(u, u^{\prime}\right)=b\left(u,(T-\alpha) u^{\prime}\right)
$$

The kernel of $b_{\alpha}$ is $\langle v\rangle$. Hence $b_{\alpha}$ descends to a non-degenerate bilinear form on the $(2 n)$ dimensional vector space $U /\langle v\rangle$. Denote by $\perp_{\alpha}$ the operation of taking the perpendicular space with respect to $b_{\alpha}$. Since $\bar{X}$ is $(n-1)$-dimensional, $b_{\alpha}$ further descends to a non-degenerate bilinear form on the 2-dimensional vector space $\bar{X}^{\perp_{\alpha}} / \bar{X}$. There are then two 1-dimensional isotropic lines. Denote by $\bar{X}_{1}$ and $\bar{X}_{2}$ their pre-images in $\bar{X}^{\perp_{\alpha}}$. The following lemma tells us how to "lift" to $L_{\{0, \ldots, 0\}}^{f, T}\left(k^{s}\right)$.

Lemma 3.9. Let $\bar{X}$ be an element of $L_{\{0,0, \ldots, 0\}}^{f /(x-\alpha)^{2}, \bar{T}}\left(k^{s}\right)$. Let $w \in U$ be an element such that $\bar{X}_{w}:=$ $\operatorname{Span}\{w+\langle v\rangle, \bar{X}\}$ is isotropic with respect to $b_{\alpha}$. Suppose, further, $b(w, v) \neq 0$. Then there exists a unique $X^{w} \in L_{\{0,0, \ldots, 0\}}^{f, T}\left(k^{s}\right)$ such that the image of $X^{w}$ under the map $U \rightarrow U /\langle v\rangle$ is $\bar{X}_{w}$.

Proof. After scaling $w$, we assume $b(w, v)=1$. Then $w-\frac{1}{2} b(w, w) v$ is the unique element of the form $w+c v$ such that $b(w+c v, w+c v)=0$. Hence by replacing $w$ with $w-\frac{1}{2} b(w, w) v$, we may 


\section{PENCILS OF QUADRICS AND HYPERELLIPTIC CURVES}

assume $b(w, w)=0$. As a consequence, any lift of $\bar{X}_{w}$ must contain $w$. The following $n$-plane is then the only lift of $\bar{X}_{w}$ that is isotropic with respect to $b$ :

$$
X^{w}=\operatorname{Span}\{w, u-b(w, u) v\}_{u+\langle v\rangle \in \bar{X}} \subset U .
$$

It is easy to see that we have $(T-\alpha) X^{w} \subset X^{w \perp}$. Since $w \notin v^{\perp}$, the $n$-plane $X^{w}$ does not contain $v$ and so contains no non-zero $T$-stable subspace. Hence $X^{w} \in L_{\{0,0, \ldots, 0\}}^{f, T}\left(k^{s}\right)$. Then

We first consider the case when $m \geqslant 3$. Let $v^{\prime \prime}$ be an element of $U$ such that $(T-\alpha) v^{\prime \prime}=v^{\prime}$.

$$
b_{\alpha}\left(v^{\prime}, v^{\prime}\right)=b\left(v^{\prime}, v\right)=b\left((T-\alpha) v^{\prime \prime}, v\right)=b\left(v^{\prime \prime},(T-\alpha) v\right)=0 .
$$

Hence we may assume, without loss of generality, $\bar{X}_{1}=\operatorname{Span}\left\{v^{\prime}+\langle v\rangle, \bar{X}\right\} \subset v^{\perp} /\langle v\rangle$. Let $w$ be an element of $U$ such that $\bar{X}_{2}=\operatorname{Span}\{w+\langle v\rangle, \bar{X}\}$. Since $\operatorname{Span}\left\{\bar{X}_{1}, \bar{X}_{2}\right\}$ has dimension $n+1$, it is not isotropic with respect to $b_{\alpha}$. Hence $b_{\alpha}\left(w, v^{\prime}\right)=b(w, v) \neq 0$. After scaling $w$, we may assume $b(w, v)=1$. Since $b\left(v^{\prime}, v\right)=0$, we cannot hope to lift $\bar{X}_{1}$ to some $X \nsubseteq v^{\perp}$. Lemma 3.9 implies that $\bar{X}_{2}$ admits a unique lift. This proves the surjectivity.

Now, suppose that $X^{\prime} \in L_{\{0, \ldots, 0\}}^{f, T}\left(k^{s}\right)$ maps to $\bar{X}$. Then the image of $X^{\prime}$ in $U /\langle v\rangle$, denoted by $X^{\prime} /\langle v\rangle$, is an $n$-plane isotropic to $b_{\alpha}$, contains $\bar{X}$, and is $b_{\alpha}$-orthogonal to $\bar{X}$. Since it does not contain $v^{\prime}+\langle v\rangle$, we conclude that $X^{\prime} /\langle v\rangle=\bar{X}_{2}$. The uniqueness part of Lemma 3.9 then shows that the fibers have size 1.

Just as in the previous reduction step, when $m \geqslant 3$, the stabilizers $J_{T}$ and $J_{\bar{T}}$ consist of elements that are represented by the same set of polynomials in $T$ and $\bar{T}$. It is clear that if $g(T)$ stabilizes $X$, then $g(\bar{T})$ stabilizes $\bar{X}$. Conversely, if $g(\bar{T})$ stabilizes $\bar{X}$, then $g(T)$ sends $X$ to another $n$-plane that also maps to $\bar{X}$. Since there is only one such $n$-plane, we conclude that $g(T)$ also stabilizes $X$. Hence $\left|\operatorname{Stab}_{J_{T}}(X)\right|=\left|\operatorname{Stab}_{\bar{T}_{\bar{T}}}(\bar{X})\right|$.

We now deal with the case $m=2$. Write $\bar{X}_{1}=\operatorname{Span}\left\{w_{1}+\langle v\rangle, \bar{X}\right\}$ and $\bar{X}_{2}=\operatorname{Span}\left\{w_{2}+\langle v\rangle, \bar{X}\right\}$ for some $w_{1}, w_{2} \in U$. We claim $w_{1} \notin v^{\perp}$ and likewise $w_{2} \notin v^{\perp}$. Suppose, for a contradiction, $w_{1} \in v^{\perp}$; then $\bar{X}_{1} \subset v^{\perp} /\langle v\rangle$. When $m=2$, the quotient $v^{\perp} /\langle v\rangle$ is the orthogonal (with respect to $b$ ) direct sum of all the generalized eigenspaces not containing $v$ and $v^{\prime}$. Since $T-\alpha$ acts invertibly on generalized eigenspaces not containing $v$ and $v^{\prime}$, we see that $b_{\alpha}$ descends to a nondegenerate bilinear form on $v^{\perp} /\langle v\rangle$. However, $\bar{X}_{1}$ is isotropic of dimension $n$ while $v^{\perp} /\langle v\rangle$ has dimension $2 n-1$, so we have a contradiction.

We may now apply Lemma 3.9 to $\bar{X}_{w_{1}}=\bar{X}_{1}$ and $\bar{X}_{w_{2}}=\bar{X}_{2}$ to obtain two unique lifts $X^{w_{1}}$ and $X^{w_{2}}$. Hence we have proved the surjectivity and showed that the fibers have size 2 .

Regarding stabilizers, we are in the situation where compared to $J_{\bar{T}}$, the group $J_{T}$ has an extra generator $h_{0}(T)=1-2 h(T) / h(\alpha)$, where $h(x)=f(x) /(x-\alpha)^{2}$. This extra generator fixes all the generalized eigenspaces not containing $v$. Hence $h_{0}(\bar{T}) \bar{X}$ equals $\bar{X}$, and a simple computation shows that $h_{0}(\bar{T}) \bar{X}$ switches $\bar{X}_{1}$ and $\overline{X_{2}}$. If $g(\bar{T})$ stabilizes $\bar{X}$, then either $g(T)$ stabilizes $X^{w_{1}}$, or it sends $X^{w_{1}}$ to $X^{w_{2}}$, in which case $g(T) h_{0}(T)$ stabilizes $X^{w_{1}}$. Therefore, the sizes of the stabilizers remain unchanged.

Corollary 3.10. We have $\left|L_{\{0,0, \ldots, 0\}}^{f, T}\left(k^{s}\right)\right|=2^{r}$, and every element has trivial stabilizer in $J_{T}$.

Proof. This follows from induction on the degree of $f$ and the classical result on generic intersection in odd dimension recalled in Section 2.1. We write out the proof slightly differently from an induction argument so we can point out the differences between the contributions coming from roots of $f$ with odd multiplicity and the contributions from roots with even multiplicity. Rewrite 


\section{WANG}

the factorization of $f(x)$ as

$$
f(x)=\prod_{i=1}^{s_{1}+1}\left(x-\beta_{i}\right)^{2 n_{i}+1} \prod_{j=1}^{s_{2}}\left(x-\beta_{j}^{\prime}\right)^{2 n_{j}^{\prime}},
$$

where each $\beta_{i}$ is a root of $f(x)$ of odd multiplicity and each $\beta_{j}^{\prime}$ is a root of even multiplicity. Since $f(x)$ has odd degree, we know $s_{1} \geqslant 0$ and $s_{1}+s_{2}=r$. Applying Proposition 3.8 repeatedly, one obtains the sequence of maps

$$
L_{\{0,0, \ldots, 0\}}^{f, T}\left(k^{s}\right) \stackrel{1 \text { to } 1}{\longrightarrow} L_{\{0,0, \ldots, 0\}}^{\prod_{i}\left(x-\beta_{i}\right) \cdot \prod_{j}\left(x-\beta_{j}^{\prime}\right)^{2}, T^{\prime}}\left(k^{s}\right) \stackrel{2^{s_{2}} \text { to } 1}{\longrightarrow} L_{\{0,0, \ldots, 0\}}^{\prod_{i}\left(x-\beta_{i}\right), T^{\prime \prime}}\left(k^{s}\right) .
$$

The last set has $2^{s_{1}}$ elements with trivial stabilizers, by the theory of generic pencils. Applying Proposition 3.8 again, one concludes that every element in $L_{\{0,0, \ldots, 0\}}^{f, T}\left(k^{s}\right)$ has trivial stabilizer as well. The above diagram shows $\left|L_{\{0,0, \ldots, 0\}}^{f, T}\left(k^{s}\right)\right|=2^{s_{1}+s_{2}}=2^{r}$.

Proof of Theorems 3.1 and 3.6. Applying Proposition 3.7 repeatedly gives a bijection

$$
L_{\left\{d_{1}, \ldots, d_{r+1}\right\}}^{f, T}\left(k^{s}\right) \underset{\delta}{\stackrel{\sim}{\longrightarrow}} L_{\{0,0, \ldots, 0\}}^{\prod_{i}\left(x-\alpha_{i}\right)^{m_{i}-2 d_{i}, T^{\prime}}}\left(k^{s}\right)
$$

such that for any $X \in L_{\left\{d_{1}, \ldots, d_{r+1}\right\}}^{f, T}\left(k^{s}\right)$,

$$
\left|\operatorname{Stab}_{J_{T}}(X)\right|=2^{a}\left|\operatorname{Stab}_{T^{\prime}}(\delta(X))\right| \text {. }
$$

This proves Theorem 3.6. The polynomial $g(x)=\prod_{i}\left(x-\alpha_{i}\right)^{m_{i}-2 d_{i}}$ has $r+1-a$ distinct roots; hence applying Corollary 3.10 to $g$ completes the proof of Theorem 3.1.

\subsection{Even dimension}

Now, suppose that $U$ has dimension $N=2 n+2$ for $n \geqslant 1$. As above, suppose that $Q=Q_{1}$ is non-degenerate, and denote by $T$ the associated self-adjoint operator on $U$. Let $\perp$ denote the operation of taking perpendicular spaces with respect to $Q$. As in Section 2.2, let $C$ be the (possibly singular) hyperelliptic curve parameterizing the rulings in the pencil. Then $C$ is isomorphic over $k$, not canonically, to the hyperelliptic curve defined by

$$
y^{2}=(-1)^{n+1} \operatorname{det}(Q) \operatorname{det}(x I-T) .
$$

The geometric genus $p_{g}$ of $C$ is defined to be the genus of its normalization $\widetilde{C}$. Let $C^{\mathrm{sm}}$ denote the smooth locus of $C$.

Lemma 3.11. Suppose $n \geqslant 0$. If $W$ is an $(n+1)$-dimensional subspace of $U \otimes k^{s}$ isotropic with respect to $Q_{1}$ and $Q_{2}$, then $W$ is $T$-stable.

Proof. Since $Q$ is non-degenerate, we have $W=W^{\perp_{Q}}$. Then $T W \subset W^{\perp_{Q}}=W$.

Proposition 3.12. The base locus $B$ contains no $\mathbb{P}^{n}$ if and only if $p_{g} \geqslant 0$. When $C$ is reducible or, equivalently, $p_{g}=-1$, the base locus $B$ contains a unique $\mathbb{P}^{n}$.

Proof. Without loss of generality, assume that $k$ is algebraically closed. Suppose that $B$ contains $\mathbb{P} W$ for some $(n+1)$-dimensional subspace $W$ of $U$. Lemma 3.11 implies that $W$ contains an eigenvector $v$ of $T$. Since $W$ is isotropic, the eigenvalue of $v$ has multiplicity at least 2 . One can now reduce the problem to $\bar{U}=v^{\perp} /\langle v\rangle$ and the $n$-dimensional subspace $\bar{W}=W /\langle v\rangle$. Apply Lemma 3.11 and reduce repeatedly until $\operatorname{dim} \bar{U}=2$ and $\operatorname{dim} \bar{W}=1$. Applying Lemma 3.11 again, we see that $\bar{T}$ has a repeated eigenvalue and hence that all the generalized eigenspaces 


\section{PENCILS OF QUADRICS AND HYPERELLIPTIC CURVES}

of $T$ have even dimension, which implies that $C$ is reducible. When $C$ is reducible, $\bar{W}$ is the unique 1-dimensional eigenspace of $\bar{T}$, thus proving uniqueness. Existence follows from running the argument backward.

Let $F_{0}$ denote the following variety over $k$ :

$$
F_{0}=\{\mathbb{P} X \mid \operatorname{dim} \mathbb{P} X=n-1, \mathbb{P} X \subset B\} .
$$

In view of the Section 3.1 and Example 2.30, we impose an open condition and look at the following variety:

$$
F=\left\{\mathbb{P} X \in F_{0} \mid \operatorname{Span}\{X, T X\} \text { has no non-zero } T \text {-stable subspace }\right\} .
$$

Lemma 3.13. Suppose $p_{g} \geqslant 0$. Then

$$
\begin{aligned}
F & =\left\{\mathbb{P} X \in F_{0} \mid X \nsubseteq v^{\perp} \text { for all singular points }[v] \in B\right\} \\
& =\left\{\mathbb{P} X \in F_{0} \mid[v] \notin \mathbb{P} X \text { for all singular points }[v] \in B\right\} .
\end{aligned}
$$

Proof. Suppose $\mathbb{P} X \in F$. Let $[v]$ be any singular point of $B$. Since $v$ is an eigenvector, $v \notin X$. Suppose, for a contradiction, $X \subset v^{\perp}$. Then $\mathbb{P}(\operatorname{Span}\{X, v\})$ is a $\mathbb{P}^{n}$ contained in $B$, contradicting Proposition 3.12. Hence $X \nsubseteq v^{\perp}$.

Conversely, suppose $\mathbb{P} X \in F_{0} \backslash F$. Then $v \in \operatorname{Span}\{X, T X\}$ for some eigenvector $v$ of $T$. Since $X$ is isotropic with respect to every quadric in the pencil, we have $v \in \operatorname{Span}\{X, T X\} \subset X^{\perp}$. Hence $X \subset v^{\perp}$.

For the second equality, first suppose $X \subset v^{\perp}$ for some singular $[v] \in B$. Suppose, for a contradiction, $v \notin X$. Then after reducing to $v^{\perp} /\langle v\rangle$, the quotient $\left(X \cap v^{\perp}\right) /\langle v\rangle$ has dimension $n$, which contradicts Proposition 3.12. Hence $v \in X$. Conversely, if $v \in X$, then $X \subset v^{\perp}$ since $X$ is isotropic.

Remark 3.14. The main reason why $F$ was defined as in (3.1) instead of the more conceptual definitions in Lemma 3.13 is that there is still some interesting geometry when $p_{g}=-1$, as we will see toward the end of the paper, in which case (3.1) is the more appropriate definition.

As in the generic case, we work toward putting a group law on the disconnected variety

$$
G=\operatorname{Pic}^{0}(C) \dot{\cup} F \dot{\cup} \operatorname{Pic}^{1}(C) \dot{\cup} F^{\prime},
$$

where $F^{\prime}$ is a copy of $F$. In what follows, we assume $p_{g} \geqslant 0$. Since the base locus contains no $\mathbb{P}^{n}$, one can define $\tau: C \times F_{0} \rightarrow F_{0}$ as in the generic case.

Lemma 3.15. The map $\tau$ restricts to a morphism $C^{\mathrm{sm}} \times F \rightarrow F$.

Proof. Recall that given a pair $(c, \mathbb{P} X) \in C^{\mathrm{sm}} \times F$, there is a unique $\mathbb{P} Y \simeq \mathbb{P}^{n}$ in the quadric and the ruling defined by $c$ such that $\mathbb{P} X \subset \mathbb{P} Y$; then $\tau(c, \mathbb{P} X)$ is the residual intersection of $\mathbb{P} Y$ with the base locus. The claim here is $\tau(c, \mathbb{P} X) \in F$. Suppose, for a contradiction, $\mathbb{P} X^{\prime}:=\tau(c, \mathbb{P} X) \in$ $F_{0} \backslash F$. Then by Lemma 3.13, there exists a singular point $[v] \in B$ such that $v \in X^{\prime}$. Since $X$ and $X^{\prime}$ intersect at codimension 1 and $v \notin X$, we see that

$$
\mathbb{P} Y=\operatorname{Span}\{\mathbb{P} X, \tau(c, \mathbb{P} X)\}=\operatorname{Span}\{\mathbb{P} X,[v]\} .
$$

Let $\alpha$ denote the eigenvalue of $v$. Then since $\mathbb{P} X$ is isotropic with respect to every quadric in the pencil and $v$ is in the kernel of the quadric $Q_{\alpha}$, we see that $\mathbb{P} Y$ lies in $Q_{\alpha}$. Hence $c=(\alpha, 0) \notin C^{\mathrm{sm}}$. We therefore have a contradiction. 


\section{WANG}

As in the generic case, one obtains an action of $C^{\text {sm }}$ on $F \dot{\cup} F^{\prime}$ :

$$
\mathbb{P} X+(c)=-\tau(\bar{c}) \mathbb{P} X, \quad-\mathbb{P} X+(c)=\tau(c) \mathbb{P} X .
$$

This action extends to an action of $\operatorname{Div}\left(C^{\mathrm{sm}}\right)$ on $F \dot{\cup} F^{\prime}$. To show that this descends to a simply transitive action of $\operatorname{Pic}^{0}(C)$, we assume $k=k^{a}$ and work over the algebraic closure. Let $v$ be an eigenvector with eigenvalue $\alpha$ of multiplicity $m \geqslant 2$. As usual, let $(\bar{U}, \bar{Q})$ denote the $2 n$ dimensional quadratic space $v^{\perp} /\langle v\rangle$, and let $\bar{T}$ denote the descent of $T$ to $\bar{U}$. Let $\bar{C}$ denote the (possibly singular) hyperelliptic curve

$$
y^{2}=\operatorname{disc}(\bar{Q}) \operatorname{det}(x I-\bar{T})=\operatorname{disc}(Q) \operatorname{det}(x I-T) /(x-\alpha)^{2} .
$$

Note that $\bar{C} \rightarrow C$ is a partial normalization of $C$. There is a natural inclusion $\iota: C^{\mathrm{sm}} \hookrightarrow \bar{C}^{\mathrm{sm}}$. Define $\bar{B}, \bar{F}$, and $\bar{F}_{0}$ analogously to $B, F$, and $F_{0}$. Let $\mathbb{P} X$ be any element of $F$. Write, as before, $\bar{X}=\left(X \cap v^{\perp}\right) /\langle v\rangle$. Lemma 3.13 implies that $\bar{X}$ has the correct dimension. It is clear then that $\bar{X} \in \bar{F}_{0}$.

Lemma 3.16. The subspace $\operatorname{Span}\{\bar{X}, \overline{T X}\}$ has no non-zero $\bar{T}$-stable subspace.

Proof. Note that this is immediate when $C$ has only nodal singularities, for this reduction step kills the $\alpha$-generalized eigenspace and leaves the rest unchanged. In general, by Lemma 3.13, it suffices to show that $\bar{X}$ does not contain any singular point of $\bar{B}$. Let $v^{\prime} \in U$ be such that (T$\alpha) v^{\prime}=v$. Suppose, for a contradiction, that $\bar{X}$ contains $v^{\prime}+\langle v\rangle$. Then $v^{\prime}+c v \in X$ for some $c \in k^{s}$. (Note that this can only happen if $m \geqslant 4$.) Hence $v=(T-\alpha)\left(v^{\prime}+c v\right) \in X^{\perp}$, contradicting $\mathbb{P} X \in F$ by Lemma 3.13. Since the reduction step does not touch all the other generalized eigenspaces, we see that $\bar{X}$ does not contain any singular point of $\bar{B}$.

Denote this reduction step by $\delta_{v}: F \rightarrow \bar{F}$. We now have the following commutative diagram:

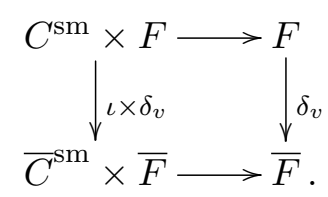

The natural map $\bar{C} \rightarrow C$ induces a map $J(C) \rightarrow J(\bar{C})$ on the Jacobians with kernel either $\mathbb{G}_{m}$ if $m=2$, or $\mathbb{G}_{a}$ if $m \geqslant 3$. We now show that $\delta_{v}$ is surjective and that the pre-image of every point is isomorphic to $\operatorname{ker}(J(C) \rightarrow J(\bar{C}))$. Let $b_{\alpha}$ denote the bilinear form $b_{\alpha}\left(u, u^{\prime}\right)=b\left(u,(T-\alpha) u^{\prime}\right)$ on $U$, and let $\perp_{\alpha}$ denote the operation of taking the perpendicular space with respect to $b_{\alpha}$. Fix any $\bar{X} \in \bar{F}$. The bilinear form $b_{\alpha}$ descends to a non-degenerate form on the $(2 n+1)$-dimensional space $U /\langle v\rangle$. Inside this space, we have

$$
\begin{aligned}
\operatorname{dim} \bar{X}^{\perp_{\alpha}} / \bar{X} & =3, \\
\operatorname{dim}\left(\bar{X}^{\perp_{\alpha}} \cap v^{\perp}\right) / \bar{X} & =2 .
\end{aligned}
$$

In other words, $b_{\alpha}$ defines a smooth conic $C_{0}$ in $\mathbb{P}^{2}=\mathbb{P}\left(\bar{X}^{\perp_{\alpha}} / \bar{X}\right)$ and $l=\mathbb{P}\left(\left(\bar{X}^{\perp_{\alpha}} \cap v^{\perp}\right) / \bar{X}\right)$ is a line intersecting the conic at either one point or two points. The same argument as in Lemma 3.9 shows that every element in $C_{0} \backslash l$ admits a unique lift to an element of $F$. Our next result gives the characterization for the intersection behavior between $C_{0}$ and $l$.

Lemma 3.17. The line $l$ intersects $C_{0}$ tangentially if and only if $m \geqslant 3$, in which case the point of intersection is $\left[v^{\prime}+\langle v\rangle+\bar{X}\right]$, where $v^{\prime} \in U$ is such that $(T-\alpha) v^{\prime}=v$. 


\section{PENCILS OF QUADRICS AND HYPERELLIPTIC CURVES}
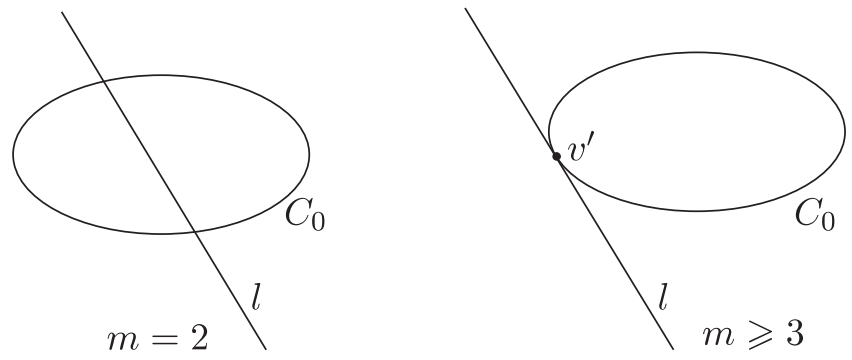

Proof. Suppose that $l$ intersects $C_{0}$ at a point $w+\langle v\rangle+\bar{X}$. To say that $l$ intersects $C_{0}$ tangentially at $w+\langle v\rangle+\bar{X}$ is equivalent to saying

$$
w+\langle v\rangle \in \bar{X}^{\perp_{\alpha}}, \quad b_{\alpha}(w, w)=0, \quad w^{\perp_{\alpha}} \cap \bar{X}^{\perp_{\alpha}}=v^{\perp} \cap \bar{X}^{\perp_{\alpha}} .
$$

Since $\left(\left(v^{\perp} \cap \bar{X}^{\perp_{\alpha}}\right)^{\perp_{\alpha}} \cap \bar{X}^{\perp_{\alpha}}\right) / \bar{X}$ is the line spanned by $v^{\prime}+\langle v\rangle+\bar{X}$, we see that the first and third conditions in (3.3) combined are equivalent to $w+\langle v\rangle+\bar{X}=v^{\prime}+\langle v\rangle+\bar{X}$ up to scaling. Finally, $b_{\alpha}\left(v^{\prime}, v^{\prime}\right)=b\left(v^{\prime}, v\right)=0$ if and only if $m \geqslant 3$.

Therefore, we have proved the following proposition.

Proposition 3.18. The map $\delta_{v}: F \rightarrow \bar{F}$ is surjective. The fibers are isomorphic to either (a conic minus a point $\simeq$ ) $\mathbb{G}_{a}$ when $m \geqslant 3$, or (a conic minus two points $\simeq$ ) $\mathbb{G}_{m}$ when $m=2$. The kernel of the map $J(C) \rightarrow J(\bar{C})$ has the same property.

One can now apply this reduction with any singular point of $\bar{B}$ and so on. For each $i$ such that $m_{i} \geqslant 2$, let $v_{i, 1}$ denote an eigenvector of $T$ with eigenvalue $\alpha_{i}$ of multiplicity $m_{i}$, and let $v_{i, j}$ be such that $\left(T-\alpha_{i}\right) v_{i, j}=v_{i, j-1}$ for $j=2, \ldots,\left\lfloor\left(m_{i}-1\right) / 2\right\rfloor$. Let $V$ denote the linear span of all such $v_{i, j}$. The above reduction will terminate at the $\left(2 p_{g}+2\right)$-dimensional vector space $\widetilde{U}=V^{\perp} / V$. The data $(Q, T)$ descends to $(\widetilde{Q}, \widetilde{T})$ on the $\left(2 p_{g}+2\right)$-dimensional vector space $\widetilde{U}=V^{\perp} / V$, with $\widetilde{T}$ regular semi-simple. Let $\widetilde{F}$ denote the variety of (linear) $p_{g}$-dimensional common isotropic subspaces $\widetilde{X}$ contained in $\widetilde{U}$. Let $\delta: F \rightarrow \widetilde{F}$ denote the composite of all the reductions. The associated smooth hyperelliptic curve $\widetilde{C}$ is the normalization of $C$. Note that if $k$ is arbitrary, then $V$ is defined over $k$ and so is the composite map $\delta$. We summarize the above discussion into the following.

TheOREm 3.19. Suppose $p_{g} \geqslant 0$ and that $k$ is algebraically closed. Then:

1. The map $\delta: F \rightarrow \widetilde{F}$ is surjective. The pre-image of every point has a filtration with $\mathbb{G}_{a^{-}}$ and $\mathbb{G}_{m}$-factors. The kernel of the natural map $J(C) \rightarrow J(\widetilde{C})$ has a filtration with the same factors.

2. There is an action of $\operatorname{Div}^{0}\left(C^{\mathrm{sm}}\right)$ on $F$ that descends to the simply transitive action of $J(\widetilde{C})$ on $\widetilde{F}$.

For the purpose of putting a natural group law on $G=\operatorname{Pic}^{0}(C) \dot{\cup} F \dot{\cup} \operatorname{Pic}^{1}(C) \dot{\cup} F^{\prime}$, it remains to show that the action of $\operatorname{Div}\left(C^{\mathrm{sm}}\right)$ on $F \dot{\cup} F^{\prime}$ descends to a simply transitive action of $\operatorname{Pic}(C) / \mathbb{Z} D_{0}$ on $F \dot{\cup} F^{\prime}$. Once again, we pass to the algebraic closure and use the same formal argument as in the generic case. We list the "non-formal" steps one needs to carry out in the regular case:

1. Prove Lemma 2.18, which allows one to define the $\infty$-minimal form of a divisor class $[D] \in$ $J(C)$ and hence a morphism $\varphi: J \rightarrow \operatorname{Aut}(F)$. Here we need to assume that $C$ has a smooth 


\section{WANG}

Weierstrass point. This is equivalent to requiring that $f(x)$ has a simple root, or equivalently, $p_{g} \geqslant 0$.

2. Show that $\varphi$ is a group homomorphism, to conclude that principal divisors supported on $C^{\mathrm{sm}}$ act trivially on $F \dot{\cup} F^{\prime}$.

3. Prove the existence part of Lemma 2.17, to conclude that the action of $J$ on $F$ is transitive.

4. Prove the uniqueness part of Lemma 2.17, to conclude that the action is simply transitive.

Lemma 2.18 still holds in the singular case because the Riemann-Roch theorem holds in the singular case [Har77, IV.1, Exercise 1.9]. Suppose that $C$ has a smooth Weierstrass point $\infty$. Note that if $C$ only has nodal singularity and $p_{g} \geqslant 0$, then $C$ has a smooth Weierstrass point. Every class $[D] \in J(C)$ has a $\infty$-minimal form $\left[D^{\prime}-r(\infty)\right]$, where $D^{\prime}$ is effective of degree $r \leqslant n$, supported on $C^{\mathrm{sm}}$, and $h^{0}\left(D^{\prime}\right)=1$. This allows us to define a morphism of varieties $\varphi: J \rightarrow \operatorname{Aut}(F)$. The image of $\varphi$ lies in a commutative subvariety of $\operatorname{Aut}(F)$.

We now specialize to the case when $C$ only has nodal singularities, so $J$ is an extension of an abelian variety $\widetilde{J}$ of dimension $p_{g}$ by a torus $S$ of dimension $n-p_{g}$.

Lemma 3.20. The map $\varphi$ is a morphism of algebraic groups.

Proof. The proof is very similar to the proof that a morphism between semi-abelian varieties mapping the identity to the identity is a group homomorphism. Without loss of generality, we may work over the algebraic closure. For any $s \in S$, its image in $\widetilde{J}$ is 0 and so by part 2 of Theorem 3.19, it acts on the fibers of the map $\delta: F \rightarrow \widetilde{F}$. For any $a \in J$, we define $\varphi_{a}: S \rightarrow \operatorname{Aut}(F)$ by

$$
\varphi_{a}(s)=\varphi(a) \varphi(s) \varphi(a s)^{-1} .
$$

Fix any $x \in F$. Let $S_{x}^{\prime}$ denote the fiber of $\delta$ over $\delta(x)$. Then $S_{x}^{\prime}$ is a torus by setting $x$ as the origin. For any $a \in J$ and any $s \in S$, we have $\delta(x)=\delta\left(\varphi_{a}(s)(x)\right)$ by part 2 of Theorem 3.19. We have then defined a map $\varphi_{a, x}: S \rightarrow S^{\prime}$ between tori sending $s$ to $\varphi_{a}(s)(x)$. Since $\varphi_{a, x}$ sends the origin of $S$ to the origin $x$ of $S_{x}^{\prime}$, we see that $\varphi_{a, x}$ is a group homomorphism. Letting $a$ vary, one obtains a map $\Phi_{x}: J \rightarrow \operatorname{Hom}\left(S, S_{x}^{\prime}\right)$ sending $a$ to $\varphi_{a, x}$. Since $J$ is connected and $\operatorname{Hom}\left(S, S_{x}^{\prime}\right)$ is discrete, $\Phi_{x}$ is constant. Hence for any $a \in J$, the map $\Phi_{x}(a)=\Phi_{x}(0)$ is the trivial map $S \rightarrow S_{x}^{\prime}$. Letting $x$ vary, we have proved that

$$
\varphi(a) \varphi(s)=\varphi(a s) \quad \forall a \in J, s \in S .
$$

Now, fix $a \in J$ and view $\varphi_{a}$ as a morphism $J \rightarrow \operatorname{Aut}(F)$. Since $\varphi_{a}$ vanishes on $S$ by (3.4), we descend $\varphi_{a}$ to a morphism $\widetilde{J} \rightarrow \operatorname{Aut}(F)$. Once again by part 2 of Theorem 3.19, fixing any $x \in F, \varphi_{a}\left(a^{\prime}\right)$ acts on the fiber over $\delta(x)$ for any $a^{\prime} \in \widetilde{J}$. Hence we have a morphism $\varphi_{a, x}: \widetilde{J} \rightarrow S_{x}^{\prime}$, sending $a^{\prime}$ to $\varphi_{a}\left(a^{\prime}\right)(x)$, which is trivial since $\widetilde{J}$ is an abelian variety and $S_{x}^{\prime}$ is a torus. Letting $x$ vary, one sees that $\varphi_{a}$ is trivial. Letting $a$ vary gives the desired result.

As in the proof of Proposition 2.15, we have shown that principal divisors supported on $C^{\mathrm{sm}}$ act trivially on $F \dot{\cup} F^{\prime}$. Next, we show the transitivity of this action. Since $\operatorname{Div}\left(C^{\mathrm{sm}}\right)$ also acts on $F_{0} \dot{\cup} F_{0}^{\prime}$ and $F \dot{\cup} F^{\prime}$ is open in $F_{0} \dot{\cup} F_{0}^{\prime}$, by taking the Zariski closure, one sees that principal divisors supported on $C^{s m}$ act trivially on $F_{0} \dot{\cup} F_{0}^{\prime}$. Since being supported on $C^{\text {sm }}$ is also an open condition, one also has that principal divisors on $C$ act trivially on $F_{0}$. The existence part of Lemma 2.17 can be applied to $F_{0}$ since the defining map $C \rightarrow \mathbb{P}^{1}$ admits no section. As a result, given $x, x^{\prime} \in F$, view them as in $F_{0}$, where there exists an effective divisor $D \in \operatorname{Div}(C)$ such that $x+D= \pm x^{\prime}$. Let $D^{\prime}$ be a divisor supported on $C^{\text {sm }}$ linearly equivalent to $D$. Since principal divisors on $C$ act trivially, $x+D^{\prime}=x+D= \pm x^{\prime}$. Transitivity then follows from the formal 


\section{PENCILS OF QUADRICS AND HYPERELLIPTIC CURVES}

argument in the proof of Proposition 2.14. Note that here, the existence of a smooth Weierstrass point is needed because we need to know that there exists $\mathbb{P} X_{0} \in F$ such that $T_{\mathbb{P} X_{0}} B \simeq \mathbb{P}^{n}$.

The uniqueness part of Lemma 2.17 also holds for $F_{0}$. The argument in [Don80] works since there is no injective map from $\mathbb{P}^{1}$ to $C$ when the arithmetic genus $n$ of $C$ is at least 1 . The same formal argument in the generic case then implies that only principal divisors act trivially. Once again, the existence of a smooth Weierstrass point $\infty$ is also needed, for we need the analog of Example 3.24 to know that there are finitely many elements of $F$ fixed by $\tau(\infty)$. By combining this with all the formal arguments in Section 2.2, we have now finished putting a natural group law on $G=\operatorname{Pic}^{0}(C) \dot{\cup} F \dot{\cup} \operatorname{Pic}^{1}(C) \dot{\cup} F^{\prime}$.

Theorem 3.21. Suppose $p_{g} \geqslant 0$ and that $C$ only has nodal singularities. Let $G$ be the disconnected variety

$$
G=\operatorname{Pic}^{0}(C) \dot{\cup} F \dot{\cup} \operatorname{Pic}^{1}(C) \dot{\cup} F^{\prime},
$$

where $F^{\prime}$ is a copy of $F$. There is a commutative algebraic group structure $+_{G}$ on $G$ over $k$ such that

1. $G^{0}=\operatorname{Pic}^{0}(C)$ with component group $G / G^{0} \simeq \mathbb{Z} / 4$;

2. $F^{\prime}$ is isomorphic to $F$ as varieties via the inversion map $-1_{G}$;

3. the group law extends that on $H=\operatorname{Pic}(C) / \mathbb{Z} D_{0} \simeq \operatorname{Pic}^{0}(C) \dot{\cup} \operatorname{Pic}^{1}(C)$, where $D_{0}$ is the hyperelliptic class;

4. the group law defines a simply transitive action of $H$ on $F \cup \dot{\cup} F^{\prime}$ extending the following action of $C^{\mathrm{sm}}$ :

$$
\mathbb{P} X+(c)=-\tau(c) \mathbb{P} X, \quad-\mathbb{P} X+(c)=\tau(\bar{c}) \mathbb{P} X,
$$

with respect to which $x+{ }_{G} x^{\prime}$, for $x, x^{\prime} \in F \dot{\cup} F^{\prime}$, is the unique divisor class sending $-x$ to $x^{\prime}$.

The following result is immediate from Theorem 3.21 and Theorem 3.19.

Corollary 3.22. Suppose $p_{g} \geqslant 0$ and that $C$ only has nodal singularities. Then the short exact sequence

$$
1 \rightarrow S \rightarrow J(C) \rightarrow J(\widetilde{C}) \rightarrow 1
$$

extends to a short exact sequence

$$
1 \rightarrow S \rightarrow G \rightarrow \widetilde{G} \rightarrow 1
$$

where $G=\operatorname{Pic}^{0}(C) \dot{\cup} F \dot{\cup} \operatorname{Pic}^{1}(C) \dot{\cup} F^{\prime}$ and $\widetilde{G}=\operatorname{Pic}^{0}(\widetilde{C}) \dot{\cup} \widetilde{F} \dot{\cup} \operatorname{Pic}^{1}(\widetilde{C}) \dot{\cup} \widetilde{F}^{\prime}$ are the corresponding disconnected groups of four components.

Now, over the algebraic closure, after identifying $F$ with $J(C)$, one can obtain a compactification of $J(C)$ by taking $F_{0}$. Recall that for any singular $[v] \in B$, we have the reduction map $\delta_{v}: F_{0} \rightarrow \bar{F}_{0}$. Note that this map might not be a morphism of varieties. The composition of all the reduction maps gives $\delta: F_{0} \rightarrow \widetilde{F} \simeq J(\widetilde{C})$. Every fiber of $\delta_{v}$ intersects $F_{0} \backslash F$ at one point, obtained by taking the pre-image of $\mathbb{P} X \in \bar{F}_{0}$ under the map $v^{\perp} \rightarrow v^{\perp} /\langle v\rangle$.

Corollary 3.23. Suppose $p_{g} \geqslant 0$ and that $C$ has only nodal singularities. Then $F_{0}$ is a compactification of $J(C)$ by adding one point to each $\mathbb{G}_{m}$-factor of the fiber over $J(\widetilde{C})$.

We expect that the condition of $C$ having only nodal singularities is unnecessary. If Theorem 3.21 is proved without this condition, then Corollary 3.23 also holds without this condition. The compactification $F_{0}$ is not smooth. 


\section{WANG}

Since multiplication by 2 and 4 are still surjective on $J$, we can lift $F$ to a torsor of $J[4]$ by taking

$$
F[4]=\left\{\mathbb{P} X \in F \mid \mathbb{P} X+_{G} \mathbb{P} X+_{G} \mathbb{P} X+_{G} \mathbb{P} X=0\right\} .
$$

When $\operatorname{Pic}^{1}(C)(k) \neq \emptyset$, the class $[F]$ is 2-torsion. For each $\left[D_{1}\right] \in \operatorname{Pic}^{1}(C)(k)$, we obtain a lift of $F$ to a torsor of $J[2]$ by taking

$$
F[2]_{\left[D_{1}\right]}=\left\{\mathbb{P} X \in F \mid \mathbb{P} X+_{G} \mathbb{P} X=\left[D_{1}\right]\right\} .
$$

For the remainder of this section, we study the special case when $\left[D_{1}\right]=(c)$ comes from a point on the curve. The situation when $c$ is a Weierstrass point is very similar to the generic case. The situation when $c$ is not a Weierstrass point has much interesting geometry which we devote most of what follows to study.

\subsubsection{Example: Smooth rational Weierstrass point}

EXAMPLE 3.24. Suppose that $C$ has a smooth rational Weierstrass point. By moving this point to $\infty$, we assume that $Q_{1}$ is singular with cone point $\left[v_{\infty}\right]$. Let $H=v_{\infty}^{\perp}{ }^{\perp} Q_{2}$ be the hyperplane in $U$ orthogonal to $v_{\infty}$ with respect to $Q_{2}$. Then $\tau(\infty)$ is induced by the linear map on $U$ that fixes $H$ and sends $v_{\infty}$ to $-v_{\infty}$. Hence

$$
F[2]_{\infty}=\{\mathbb{P} X \in F \mid \mathbb{P} X \subset B \cap \mathbb{P} H\} .
$$

Just as in the generic case, when restricted to the $(2 n+1)$-dimensional vector space $H$, the quadrics $Q_{1}$ and $Q_{2}$ span a regular pencil $\mathcal{L}_{H}$. Moreover, $Q_{1 \mid H}$ is non-degenerate and $T_{\mid H}$ restricts to the self-adjoint operator on $H$ associated to the pencil $\mathcal{L}_{H}$, as defined in (2.1). The right-hand side of (3.5) is precisely $L_{\{0,0, \ldots, 0\}}^{f, T_{\mid H}}$ as defined in the odd-dimension case. Now, $J[2]$ acts on $F[2]_{\infty}$ via the action of $J$ and on $L_{\{0,0, \ldots, 0\}}^{f, T_{\mid H}}$ via the identification $J[2] \simeq \operatorname{Stab}_{\mathrm{PO}\left(H, Q_{1 \mid H}\right)}(T)$. As in the generic case, these two actions coincide.

\subsubsection{Example: Rational non-Weierstrass point}

EXAMPLE 3.25. Suppose that $C$ has a rational non-Weierstrass point or, equivalently, that $\mathcal{L}$ has a rational quadric with discriminant 1 . By moving this point to infinity, we assume that $Q_{1}$ has discriminant 1 . The two rulings of $Q_{1}$ are then defined over $k$. Let $Y_{0}$ denote one of the rulings, and let $\infty \in C(k)$ denote the point corresponding to the quadric $Q_{1}$ and the ruling $Y_{0}$. Denote by $\infty^{\prime}$ the conjugate of $\infty$ under the hyperelliptic involution. As in the generic case, we have

$$
F[2]_{\infty}=\{\mathbb{P} X \in F \mid \mathbb{P} X=\tau(\infty) \mathbb{P} X\}=\left\{\mathbb{P} X \in F \mid \operatorname{Span}\{\mathbb{P} X, \mathbb{P}(T X)\} \sim Y_{0}\right\} .
$$

The latter condition means that $\operatorname{Span}\{\mathbb{P} X, \mathbb{P}(T X)\} \simeq \mathbb{P}^{n}$ is contained in $Q_{1}$ in the ruling $Y_{0}$.

Fix the monic polynomial $f(x)$ of degree $2 n+2$ splitting completely over $k^{s}$ and the quadratic form $Q=Q_{1}$ of discriminant 1 . For every field $k^{\prime}$ containing $k$, define

$$
V_{f}\left(k^{\prime}\right)=\left\{T: U \otimes k^{\prime} \rightarrow U \otimes k^{\prime} \mid T \text { is self-adjoint and regular with minimal polynomial } f(x)\right\} \text {. }
$$

For every field $k^{\prime}$ containing $k$ and every $T \in V_{f}\left(k^{\prime}\right)$, let $W_{T}\left(k^{\prime}\right)$ denote the set of (linear) $n$ dimensional $k^{\prime}$-subspaces $X$ of $U \otimes k^{\prime}$ such that $\operatorname{Span}\{X, T X\} \sim Y_{0}$. That is, the linear space $\operatorname{Span}\{X, T X\}$ is an $(n+1)$-dimensional isotropic subspace with respect to $Q$ that lies inside the ruling $Y_{0}$. As before, we define

$$
W_{f}\left(k^{\prime}\right)=\left\{(T, X) \mid T \in V_{f}\left(k^{\prime}\right), X \in W_{T}\left(k^{\prime}\right)\right\} .
$$




\section{PENCILS OF QUADRICS AND HYPERELLIPTIC CURVES}

There is a Galois-equivariant action of $\operatorname{PSO}(U, Q)=\mathrm{SO}(U, Q) /( \pm 1)$ on $W_{f}$ :

$$
g \cdot(T, X)=\left(g T g^{-1}, g X\right) \text {. }
$$

Recall that $U \otimes k^{s}$ breaks up as the orthogonal direct sum of generalized eigenspaces $U_{i, T}$ of $T$ of dimension $m_{i}$. For any linear space $X$, we defined $\operatorname{dim}_{i, T}(X)$ to be the dimension of the maximal $T$-stable subspace in $\left(X \otimes k^{s}\right) \cap U_{i, T}$. For any sequence of integers $d_{1}, \ldots, d_{r+1}$ such that $0 \leqslant d_{i} \leqslant m_{i} / 2$, we defined for any field $k^{\prime}$ containing $k$,

$$
L_{\left\{d_{1}, \ldots, d_{r+1}\right\}}^{f, T}\left(k^{\prime}\right)=\left\{X \in W_{T}\left(k^{\prime}\right) \mid \operatorname{dim}_{i, T}(X)=d_{i}\right\} .
$$

In view of the definition of $F$, we also define,

$$
\begin{aligned}
L_{\left\{d_{1}, \ldots, d_{r+1}\right\}}^{f f, T}\left(k^{\prime}\right) & =\left\{X \in W_{T}\left(k^{\prime}\right) \mid \operatorname{dim}_{i, T}(\operatorname{Span}\{X, T X\})=d_{i}\right\}, \\
W_{\left\{d_{1}, \ldots, d_{r+1}\right\}}^{\prime f}\left(k^{\prime}\right) & =\left\{(T, X) \mid T \in V_{f}\left(k^{\prime}\right), X \in L_{\left\{d_{1}, \ldots, d_{r+1}\right\}}^{\prime f, T}\left(k^{\prime}\right)\right\} .
\end{aligned}
$$

Note that when all the $d_{i}$ are 0 , we have $L_{\{0, \ldots, 0\}}^{\prime f, T}\left(k^{\prime}\right)=F[2]_{\infty}\left(k^{\prime}\right)$.

We make no assumption on the reducibility of the associated hyperelliptic curve $C$ but assume instead a weaker condition,

$$
d_{1}+\cdots+d_{r+1}<n+1=\operatorname{dim} \operatorname{Span}\{X, T X\} .
$$

Then condition (3.6) holds if and only if $\operatorname{Span}\{X, T X\}$ is not $T$-stable. Let $s_{1}$ denote the number of roots of $f$ with odd multiplicity. Then the maximum $d_{1}+\cdots+d_{r+1}$ could reach is $n+1-s_{1} / 2$. If (3.6) fails, then we must have $s_{1}=0$ and hence $C$ is reducible. If one uses $L^{f, T}$ instead of $L^{\prime f, T}$ or if one does not assume (3.6), then there will be infinitely many choices for $X$ when $C$ is reducible. See Examples 3.37 and 3.38.

As one would expect from the odd case, the main theorems we are heading toward are the following.

TheOrem 3.26. Suppose $d_{1}+\cdots+d_{r+1}<n+1$. Let a denote the number of $d_{i}$ equal to $m_{i} / 2$. Then

$$
\left|L_{\left\{d_{1}, \ldots, d_{r+1}\right\}}^{\prime f, T}\left(k^{s}\right)\right|=2^{r} / 2^{a}
$$

Theorem 3.27. Suppose $d_{1}+\cdots+d_{r+1}<n+1$. Then $\operatorname{PSO}(U, Q)\left(k^{s}\right)$ acts on $W_{\left\{d_{1}, \ldots, d_{r+1}\right\}}^{\prime f}\left(k^{s}\right)$ simply transitively if $a=0$ and transitively if $a>0$.

We begin by studying the conjugation action of $\operatorname{PSO}(U, Q)$ on $V_{f}$. The proof of the following result is identical to that of Proposition 3.4.

Proposition 3.28. The action of $\operatorname{PSO}(U, Q)$ on $V_{f}$ has a unique geometric orbit. For any $T \in V_{f}\left(k^{\prime}\right)$ defined over some field $k^{\prime}$ over $k$, its stabilizer scheme $\operatorname{Stab}(T)$ is isomorphic to $\left(\operatorname{Res}_{L^{\prime} / k^{\prime}} \mu_{2}\right)_{N=1} / \mu_{2} \simeq J[2]$ as group schemes over $k^{\prime}$, where $L^{\prime}=k^{\prime}[x] / f(x)$. In particular, $\operatorname{Stab}_{\mathrm{PSO}(U, Q)}(T)\left(k^{s}\right)$ is an elementary abelian 2-group of order $2^{r}$.

Remark 3.29. A more explicit description for the stabilizer in terms of polynomials in $T$ is almost identical to the odd case as given in Remark 3.5. For each $i=1, \ldots, r+1$, define 


\section{WANG}

$h_{i}^{T}(x)=f(x) /\left(x-\alpha_{i}\right)^{m_{i}}$. Then

$$
\begin{aligned}
\mu_{2}\left(k^{s}[T]^{\times}\right) & =\left\{\prod_{i \in I}\left(1-2 \frac{h_{i}^{T}(T)}{h_{i}^{T}\left(\alpha_{i}\right)}\right)\right\}_{I \subset\{1, \ldots, r+1\}, 2|| I \mid} \\
& =\left\{1-2 \sum_{i \in I} \frac{h_{i}^{T}(T)}{h_{i}^{T}\left(\alpha_{i}\right)}\right\}_{I \subset\{1, \ldots, r+1\}, 2|| I \mid}
\end{aligned}
$$

For any $T \in V_{f}\left(k^{s}\right)$, its stabilizer $J_{T}$ in $\operatorname{PSO}(U, Q)\left(k^{s}\right)$ acts on $L_{\left\{d_{1}, \ldots, d_{r+1}\right\}}^{\prime f, T}\left(k^{s}\right)$. Then just as Theorem 3.2 follows from Theorems 3.1 and 3.6, Theorem 3.27 follows from Theorems 3.26 and 3.30 .

Theorem 3.30. Suppose $d_{1}+\cdots+d_{r+1}<n+1$. Let a denote the number of $d_{i}$ equal to $m_{i} / 2$. Then for any $X \in L_{\left\{d_{1}, \ldots, d_{r+1}\right\}}^{\prime f, T}\left(k^{s}\right)$,

$$
\left|\operatorname{Stab}_{J_{T}}(X)\right|=2^{a}
$$

We will prove Theorems 3.26 and 3.30 via the same series of reductions as in the odddimension case.

In what follows, it turns out that one should forget about the rulings in the following reductions. Namely, consider instead

$$
W_{T}^{*}\left(k^{s}\right)=\left\{X \in \operatorname{Gr}\left(n, U \otimes k^{s}\right) \mid \operatorname{Span}\{X, T X\} \text { is }(n+1) \text {-dimensional and isotropic }\right\} .
$$

Observe that $W_{T}^{*}\left(k^{s}\right)$ can be divided into two components, one of which is $W_{T}\left(k^{s}\right)$, corresponding to which ruling $\operatorname{Span}\{X, T X\}$ lies in. The two components are in bijection with each other via an element $g_{0}$ in $\operatorname{Stab}_{\mathrm{PO}}(T)\left(k^{s}\right)$ that does not lie in $\operatorname{Stab}_{\mathrm{PSO}}(T)\left(k^{s}\right)$. One similarly defines $L_{\left\{d_{1}, \ldots, d_{r+1}\right\}}^{f, T, *}\left(k^{s}\right)$ and $L_{\left\{d_{1}, \ldots, d_{r+1}\right\}}^{f, T, *}\left(k^{s}\right)$.

Once again, most of the following results are proved by hardcore linear algebra. We recommend the interested readers to work out the proofs themselves.

Reduction on $d_{1}, \ldots, d_{r+1}$. Suppose $X \in L_{\left\{d_{1}, \ldots, d_{r+1}\right\}}^{f, T, *}\left(k^{s}\right)$ with $d_{i} \geqslant 1$. Let $v_{i}$ denote an eigenvector of $T$ corresponding to $\alpha_{i}$. Since $T$ is regular, $v_{i}$ is unique up to scaling. The assumption $d_{i} \geqslant 1$ implies $v_{i} \in \operatorname{Span}\{X, T X\}$. Hence

$$
X \subset \operatorname{Span}\{X, T X\}^{\perp} \subset v_{i}^{\perp} .
$$

Let $b$ denote the bilinear form associated to $Q$. As before, the data $(Q, T)$ descends to $\left(\bar{Q}_{i}, \bar{T}_{i}\right)$ on $\bar{U}_{i}:=v_{i}^{\perp} /\left\langle v_{i}\right\rangle$ and $\bar{T}_{i}$ is regular with characteristic polynomial $f(x) /\left(x-\alpha_{i}\right)^{2}$. Let $\bar{X}_{i}$ denote the image of $X$ in $\bar{U}_{i}$. Then $\operatorname{Span}\left\{\bar{X}_{i}, \bar{T}_{i} \bar{X}_{i}\right\}$ is an isotropic $n$-plane with respect to $\bar{Q}_{i}$, and the dimensions of the maximal $\bar{T}_{i}$-stable subspaces in the intersection of $\operatorname{Span}\left\{\bar{X}_{i}, \bar{T}_{i} \bar{X}_{i}\right\}$ with the generalized eigenspaces are $d_{1}, \ldots, d_{i}-1, \ldots, d_{r+1}$. Suppose, for a contradiction, $v_{i} \notin X$. Then $\operatorname{Span}\left\{X, v_{i}\right\}$ is an isotropic $(n+1)$-plane. Hence $\operatorname{Span}\{X, T X\}$ is either $\operatorname{Span}\left\{X, v_{i}\right\}$ or $g_{0} \operatorname{Span}\left\{X, v_{i}\right\}$, both of which are $T$-stable, violating condition (3.6). Hence $v_{i} \in X$ and so $\bar{X}_{i}$ is $(n-1)$-dimensional. We denote this reduction step by

$$
L_{\left\{d_{1}, \ldots, d_{r+1}\right\}}^{\prime f, T, *}\left(k^{s}\right) \underset{\delta_{i}}{\stackrel{\sim}{\longrightarrow}} L_{\left\{d_{1}, \ldots, d_{i}-1, \ldots, d_{r+1}\right\}}^{\prime f /\left(x-\alpha_{i}\right)^{2}, \bar{T}_{i}, *}\left(k^{s}\right) .
$$

The map $\delta_{i}$ is bijective, its inverse is given by taking the pre-image of the projection map $H_{i} \rightarrow \bar{U}_{i}$. 


\section{PENCILS OF QUADRICS AND HYPERELLIPTIC CURVES}

The stabilizers are affected in the same manner as in the odd case. We summarize this reduction step in the following proposition.

Proposition 3.31. Suppose $d_{i} \geqslant 1$; then there is a bijection

$$
\delta_{i}: L_{\left\{d_{1}, \ldots, d_{r+1}\right\}}^{\prime f, T, *}\left(k^{s}\right) \stackrel{\sim}{\longrightarrow} L_{\left\{d_{1}, \ldots, d_{i}-1, \ldots, d_{r+1}\right\}}^{\prime f /\left(x-\alpha_{i}\right)^{2}, \bar{T}_{i}, *}\left(k^{s}\right) .
$$

When $m_{i} \geqslant 3$, the map $\delta_{i}$ does not change the sizes of the stabilizers. When $m_{i}=2$, the map $\delta_{i}$ lowers the sizes of the stabilizers by a factor of 2 .

The map $\delta_{i}$ can be described projectively as intersecting the quadric defined by $Q$ with the tangent plane to $v_{i}$, then projecting away from $v_{i}$. Such an operation does not preserve the rulings. Two (projective) $n$-planes in $Q$ lying in the same ruling could be sent to different rulings via this procedure. For example, take a smooth quadric in $\mathbb{P}^{7}$ and two 3-planes $Y_{1}$ and $Y_{2}$ on the quadric intersecting at a line. Then these two 3-planes lie on the same ruling. If the tangent plane to $v$ contains this line, then the images of $Y_{1}$ and $Y_{2}$ lie in different rulings since their intersection codimension is 1 . If the tangent plane to $v_{i}$ meets this line at a point, then the images of $Y_{1}$ and $Y_{2}$ lie in the same ruling as their intersection codimension is 2. Similar examples can be written down when $Y_{1}$ and $Y_{2}$ lie on different rulings. This is the main reason why forgetting the rulings is more convenient.

Reduction on $f$. By the above reduction step, it remains to study $L_{\{0,0, \ldots, 0\}}^{\prime f, T, *}\left(k^{s}\right)$. Let $X \in$ $W_{T}^{*}\left(k^{s}\right)$ be arbitrary. By Lemma 3.13, the following conditions are equivalent:

1. The linear space $\operatorname{Span}\{X, T X\}$ contains no $T$-stable subspace.

2. We have $v \notin X$ for any eigenvector $v$ whose eigenvalue has multiplicity at least 2 .

3. We have $X \nsubseteq v^{\perp}$ for any eigenvector $v$ whose eigenvalue has multiplicity at least 2 .

The arguments in the odd-dimension case rely much on the second and the third conditions. Since they are equivalent to the first condition, we see that these arguments carry through immediately and will not repeat them. The main difference to the odd-dimension case is due to dimension reasons which one handles by replacing certain $X$ (and $\bar{X}$ ) by $\operatorname{Span}\{X, T X\}$ (and $\operatorname{Span}\{\bar{X}, \overline{T X}\})$.

Suppose that $\alpha$ is a root of $f$ of multiplicity $m \geqslant 2$. Let $X \in L_{\{0,0, \ldots, 0\}}^{f f, T}\left(k^{s}\right)$ be arbitrary. Let $v$ denote an eigenvector of $T$ with eigenvalue $\alpha$. Then, as before, the non-degenerate quadratic form $Q$ descends to a non-degenerate quadratic form $\bar{Q}$ on $\bar{U}=v^{\perp} /\langle v\rangle$ and $T$ descends to a regular self-adjoint operator $\bar{T}$ on $\bar{U}$ with characteristic polynomial $f(x) /(x-\alpha)^{2}$. Let $\bar{X}$ denote the image of $X \cap v^{\perp}$ under the quotient map $U \rightarrow U /\langle v\rangle$.

Proposition 3.32. The above map sending $X$ to $\bar{X}$ defines a surjection

$$
L_{\{0,0, \ldots, 0\}}^{\prime f, T, *}\left(k^{s}\right) \longrightarrow L_{\{0,0, \ldots, 0\}}^{\prime f /(x-\alpha)^{2}, \bar{T}, *}\left(k^{s}\right) .
$$

This map is bijective if $m>2$ and is two-to-one if $m=2$. In both cases,

$$
\left|\operatorname{Stab}_{J_{T}}(X)\right|=\left|\operatorname{Stab}_{\bar{T}}(\bar{X})\right| \quad \text { for any } X \in L_{\{0, \ldots, 0\}}^{f f, T}\left(k^{s}\right) .
$$

Proof. Take any $\bar{X} \in L_{\{0,0, \ldots, 0\}}^{\prime f /(x-\alpha)^{2}, \bar{T}, *}\left(k^{s}\right)$. As in the odd-dimension case, let $b_{\alpha}$ denote the bilinear form

$$
b_{\alpha}\left(u, u^{\prime}\right)=b\left(u,(T-\alpha) u^{\prime}\right),
$$




\section{WANG}

where $b$ denotes the bilinear form associated to $Q$. Then $b_{\alpha}$ descends to a non-degenerate bilinear form on the $(2 n+1)$-dimensional vector space $U /\langle v\rangle$. Denote by $\perp_{\alpha}$ the perpendicular space with respect to $b_{\alpha}$. We claim that $b_{\alpha}$ descends to a non-degenerate bilinear form on the 2-dimensional vector space $\bar{Y}=\operatorname{Span}\{\bar{X}, \overline{T X}\}^{\perp_{\alpha}} / \bar{X}$. Indeed, we know a priori that $b_{\alpha}$ descends to a nondegenerate form on the 3-dimensional space $\bar{X}^{\perp_{\alpha}} / \bar{X}$. We claim

$$
\bar{X}^{\perp_{\alpha}}=\operatorname{Span}\left\{\operatorname{Span}\{\bar{X}, \overline{T X}\}^{\perp_{\alpha}}, \overline{T X}\right\} .
$$

Indeed, since $\operatorname{Span}\{\bar{X}, \overline{T X}\}$ is isotropic with respect to $b$, we have $\overline{T X} \subset \bar{X}^{\perp_{\alpha}}$. It remains to show $\overline{T X} \nsubseteq \operatorname{Span}\{\bar{X}, \overline{T X}\}^{\perp_{\alpha}}$. In other words, we need to show that $\operatorname{Span}\{\bar{X}, \overline{T X}\}$ is not isotropic with respect to $b_{\alpha}$. Suppose, for a contradiction, that $\operatorname{Span}\{\bar{X}, \overline{T X}\}$ is isotropic with respect to $b_{\alpha}$. Then inside $\bar{U}$,

$$
\bar{T}^{2} \bar{X} \subset \operatorname{Span}\{\bar{X}, \overline{T X}\}^{\perp}=\operatorname{Span}\{\bar{X}, \overline{T X}\} .
$$

Hence the entire $\operatorname{Span}\{\bar{X}, \overline{T X}\}$ is $\bar{T}$-stable. This gives a contradiction.

Now, take any $w \in \operatorname{Span}\{\bar{X}, \overline{T X}\}^{\perp_{\alpha}}$. One can first find some $w^{\prime} \in \bar{X}^{\perp_{\alpha}}$ such that $b_{\alpha}\left(w, w^{\prime}\right)$ is non-zero. Let $u^{\prime}$ be an element of $\overline{T X}$ such that $w^{\prime}-u^{\prime} \in \operatorname{Span}\{\bar{X}, \overline{T X}\}^{\perp_{\alpha}}$. Then $b_{\alpha}\left(w, w^{\prime}-u^{\prime}\right)=$ $b_{\alpha}\left(w, w^{\prime}\right) \neq 0$. Hence we have shown that $b_{\alpha}$ is non-degenerate on the 2-dimensional space $\bar{Y}$. There are then two 1-dimensional isotropic lines. Denote by $\bar{X}_{1}$ and $\bar{X}_{2}$ their pre-images in $\operatorname{Span}\{\bar{X}, \overline{T X}\}^{\perp_{\alpha}}$.

The case $m \geqslant 3$ is identical to the odd-dimension case: one of $\bar{X}_{1}$ and $\bar{X}_{2}$ does not lift while the other one lifts uniquely to some $X^{w}$. The condition that $\bar{X}_{1}$ and $\bar{X}_{2}$ lie inside $\operatorname{Span}\{\bar{X}, \overline{T X}\}^{\perp_{\alpha}}$ ensures that $\operatorname{Span}\left\{X^{w}, T X^{w}\right\}$ is isotropic with respect to $Q$.

Now, suppose $m=2$. Write $\bar{X}_{1}=\operatorname{Span}\left\{w_{1}+\langle v\rangle, \bar{X}\right\}$ and $\bar{X}_{2}=\operatorname{Span}\left\{w_{2}+\langle v\rangle, \bar{X}\right\}$. The only difference to the odd-dimension case is the proof of $w_{1} \notin v^{\perp}$. Suppose, for a contradiction, $w_{1} \in$ $v^{\perp}$. Since $\operatorname{Span}\{\bar{X}, \overline{T X}\}$ is not isotropic with respect to $b_{\alpha}$, we see that we have $\operatorname{Span}\{\bar{X}, \overline{T X}\} \neq$ $\operatorname{Span}\left\{\bar{X}, w_{1}+\langle v\rangle\right\}$ and so $\operatorname{Span}\{\bar{X}, \overline{T X}, w+\langle v\rangle\}$ is an $(n+1)$-dimensional subspace of $v^{\perp} /\langle v\rangle$. As in the odd case, $b_{\alpha}$ is non-degenerate on $v^{\perp} /\langle v\rangle$ because $T-\alpha$ acts invertibly on $v^{\perp} /\langle v\rangle$. However, taking $\perp_{\alpha}$ inside $v^{\perp} /\langle v\rangle$, we see that we have

$$
\operatorname{Span}\left\{\bar{X}, \overline{T X}, w_{1}+\langle v\rangle\right\}^{\perp_{\alpha}} \supset \operatorname{Span}\left\{w_{1}+\langle v\rangle, \bar{X}\right\} .
$$

The left-hand side has dimension $n-1$ while the right-hand side has dimension $n$, giving a contradiction. The rest of the argument is identical to the odd-dimension case: $\bar{X}_{1}$ and $\bar{X}_{2}$ each has a unique lift.

Stabilizers behave in the same way as in the odd-dimension case.

Proposition 3.33. We have $\left|L_{\{0,0, \ldots, 0\}}^{\prime f, T, *}\left(k^{s}\right)\right|=2^{r+1}$, and every element has trivial stabilizer in $J_{T}$.

Proof. Apply the reduction steps like in the odd-dimension case. There are now five base cases, which we illustrate below as examples.

EXAMPLE 3.34 (Generic case). Suppose that reduction terminates with $f(x)=\prod_{i=1}^{r+1}\left(x-\alpha_{i}\right)$ with $r \geqslant 3$. In this case, one can apply the theory for the non-singular case discussed in Example 2.30 and obtain $\left|L_{\{0,0, \ldots, 0\}}^{\prime f, T, *}\left(k^{s}\right)\right|=2^{r+1}$.

EXAMPLE 3.35. Suppose that reduction terminates with $f(x)=(x-\alpha)(x-\beta)(x-\gamma)^{2}$. If one tries to apply reduction again on $\gamma$, then $\bar{X}$ becomes 0 -dimensional. Let $u, v, w_{1}$ denote eigenvectors 


\section{PENCILS OF QUADRICS AND HYPERELLIPTIC CURVES}

of $T$ with eigenvalues $\alpha, \beta, \gamma$, respectively, and let $w_{2}$ be such that $(T-\gamma) w_{2}=w_{1}$. We seek coefficients $c_{1}, \ldots, c_{4}$ such that $X=\left\langle c_{1} u+c_{2} v+c_{3} w_{1}+c_{4} w_{2}\right\rangle$ lies in $L_{0,0,0}^{\prime f, T, *}\left(k^{s}\right)$. Set

$$
\Omega_{1}=b(u, u) \neq 0, \quad \Omega_{2}=b(v, v) \neq 0, \quad \Gamma_{3}=b\left(w_{1}, w_{2}\right) \neq 0, \quad \Gamma_{4}=b\left(w_{2}, w_{2}\right) .
$$

Then the condition that $\operatorname{Span}\{X, T X\}$ is an isotropic 2-plane becomes

$$
\left(\begin{array}{cccc}
\Omega_{1} & \Omega_{2} & \Gamma_{3} & \Gamma_{4} \\
(\gamma-\alpha) \Omega_{1} & (\gamma-\beta) \Omega_{2} & 0 & \Gamma_{3} \\
(\gamma-\alpha)^{2} \Omega_{1} & (\gamma-\beta)^{2} \Omega_{2} & 0 & 0
\end{array}\right)\left(\begin{array}{c}
c_{1}^{2} \\
c_{2}^{2} \\
2 c_{3} c_{4} \\
c_{4}^{2}
\end{array}\right)=\left(\begin{array}{l}
0 \\
0 \\
0 \\
0
\end{array}\right)
$$

Since $\Gamma_{3}, \Omega_{1}, \Omega_{2}$ are non-zero, the above matrix has a 1-dimensional kernel. Moreover, if any one of $c_{1}, c_{2}, c_{4}$ is zero, then they are all zero and $X$ is of the form $\left\langle c_{3} w_{1}\right\rangle$, which does not lie in $L_{0,0,0}^{\prime f, T, *}\left(k^{s}\right)$. Now, given non-zero $c_{1}, c_{2}, c_{4}$ obtained by taking a non-zero vector in the 1-dimensional kernel and taking square roots of the corresponding entries, there is a unique solution for $c_{3}$. Therefore, there are $8=2^{3}$ choices for $X$ depending on which square roots one chooses for $c_{1}, c_{2}, c_{4}$.

EXAMPLE 3.36. Suppose that reduction terminates with $f(x)=(x-\alpha)^{3}(x-\beta)$. Let $u_{1}$ and $v$ denote eigenvectors of $T$ with eigenvalues $\alpha$ and $\beta$, respectively, and let $u_{2}$ and $u_{3}$ be such that $(T-\alpha)^{2} u_{3}=(T-\alpha) u_{2}=u_{1}$. We seek coefficients $c_{1}, \ldots, c_{4}$ such that $X=\left\langle c_{1} u_{1}+c_{2} u_{2}+c_{3} u_{3}+c_{4} v\right\rangle$ lies in $L_{0,0}^{\prime f, T, *}\left(k^{s}\right)$. Set

$$
\Omega=b(v, v) \neq 0, \quad \Gamma_{4}=b\left(u_{1}, u_{3}\right)=b\left(u_{2}, u_{2}\right) \neq 0, \quad \Gamma_{5}=b\left(u_{2}, u_{3}\right), \quad \Gamma_{6}=b\left(u_{3}, u_{3}\right) .
$$

Then the condition that $\operatorname{Span}\{X, T X\}$ is an isotropic 2-plane becomes

$$
\left(\begin{array}{cccc}
\Gamma_{4} & \Gamma_{5} & \Gamma_{6} & \Omega \\
0 & \Gamma_{4} & \Gamma_{5} & (\beta-\alpha) \Omega \\
0 & 0 & \Gamma_{4} & (\beta-\alpha)^{2} \Omega
\end{array}\right)\left(\begin{array}{c}
c_{2}^{2}+2 c_{1} c_{3} \\
2 c_{2} c_{3} \\
c_{3}^{2} \\
c_{4}^{2}
\end{array}\right)=\left(\begin{array}{l}
0 \\
0 \\
0 \\
0
\end{array}\right)
$$

Since $\Gamma_{4}$ and $\Omega$ are non-zero, the above matrix has a 1-dimensional kernel, and if any one of $c_{2}, c_{3}$, $c_{4}$ is zero, then all of them are zero and $X$ is of the form $\left\langle c_{1} u_{1}\right\rangle$, which does not lie in $L_{0,0}^{\prime f, T, *}\left(k^{s}\right)$. Now, given non-zero $c_{3}$ and $c_{4}$ obtained by taking a non-zero vector in the 1-dimensional kernel and taking square roots of the corresponding entries, there is a unique solution for $c_{1}$ and $c_{2}$. Therefore, there are $4=2^{2}$ choices for $X$ depending on which square roots one chooses for $c_{3}$ and $c_{4}$.

EXAmPLE 3.37. Suppose that reduction terminates with $f(x)=(x-\alpha)^{2}(x-\beta)^{2}$. Let $u_{1}$ and $v_{1}$ denote eigenvectors of $T$ with eigenvalues $\alpha$ and $\beta$, respectively, and let $u_{2}$ and $v_{2}$ be such that $(T-\alpha) u_{2}=u_{1}$ and $(T-\beta) v_{2}=v_{1}$. We seek coefficients $c_{1}, \ldots, c_{4}$ such that $X=\left\langle c_{1} u_{1}+c_{2} u_{2}+\right.$ $\left.c_{3} v_{1}+c_{4} v_{2}\right\rangle$ lies in $L_{0,0}^{\prime f, T, *}\left(k^{s}\right)$. Set

$$
\Gamma_{3}=b\left(u_{1}, u_{2}\right) \neq 0, \quad \Gamma_{4}=b\left(u_{2}, u_{2}\right), \quad \Omega_{3}=b\left(v_{1}, v_{2}\right) \neq 0, \quad \Omega_{4}=b\left(v_{2}, v_{2}\right) .
$$

Then the condition that $\operatorname{Span}\{X, T X\}$ is an isotropic 2-plane becomes

$$
\left(\begin{array}{cccc}
\Gamma_{3} & \Gamma_{4} & \Omega_{3} & \Omega_{4} \\
0 & \Gamma_{3} & (\beta-\alpha) \Omega_{3} & \Omega_{3}+(\beta-\alpha) \Omega_{4} \\
0 & 0 & (\beta-\alpha)^{2} \Omega_{3} & 2(\beta-\alpha) \Omega_{3}+(\beta-\alpha)^{2} \Omega_{4}
\end{array}\right)\left(\begin{array}{c}
2 c_{1} c_{2} \\
c_{2}^{2} \\
2 c_{3} c_{4} \\
c_{4}^{2}
\end{array}\right)=\left(\begin{array}{l}
0 \\
0 \\
0 \\
0
\end{array}\right)
$$




\section{WANG}

Since $\Gamma_{3}$ and $\Omega_{3}$ are non-zero, the above matrix has a 1-dimensional kernel. If any one of $c_{2}$ and $c_{4}$ is zero, then both of them are zero and $X$ is of the form $\left\langle c_{1} u_{1}+c_{3} v_{1}\right\rangle$. In this case, $\operatorname{Span}\{X, T X\}$ contains either $\left\langle u_{1}\right\rangle$ or $\left\langle v_{1}\right\rangle$, both of which are $T$-stable, thereby forcing $X \notin L_{0,0}^{\prime f, *}\left(k^{s}\right)$. Note that if $c_{1}$ and $c_{3}$ are both non-zero, then $X$ satisfies the weaker condition that $X$ contains no non-zero $T$-stable subspace while $X \in L_{1,1}^{f, T, *}\left(k^{s}\right)$ violates condition (3.6). It is clear that there are infinitely many such $X$.

Now, given non-zero $c_{2}$ and $c_{4}$ obtained by taking a non-zero vector in the 1-dimensional kernel and taking square roots of the corresponding entries, there is a unique solution for $c_{1}$ and $c_{3}$. Therefore, there are $4=2^{2}$ choices for $X$ depending on which square roots one chooses for $c_{2}$ and $c_{4}$.

EXAMPLE 3.38. Suppose that reduction terminates with $f(x)=(x-\alpha)^{4}$. Let $u_{1}$ denote an eigenvector of $T$ with eigenvalue $\alpha$, and let $u_{2}, u_{3}, u_{4}$ be such that

$$
(T-\alpha)^{3} u_{4}=(T-\alpha)^{2} u_{3}=(T-\alpha) u_{2}=u_{1} .
$$

We seek coefficients $c_{1}, \ldots, c_{4}$ such that $X=\left\langle c_{1} u_{1}+c_{2} u_{2}+c_{3} u_{3}+c_{4} u_{4}\right\rangle$ lies in $L_{0}^{f, T, *}\left(k^{s}\right)$. Set

$$
\Gamma_{5}=b\left(u_{1}, u_{4}\right) \neq 0, \quad \Gamma_{6}=b\left(u_{2}, u_{4}\right)=b\left(u_{3}, u_{3}\right), \quad \Gamma_{7}=b\left(u_{3}, u_{4}\right), \quad \Gamma_{8}=b\left(u_{4}, u_{4}\right) .
$$

Then the condition that $\operatorname{Span}\{X, T X\}$ is an isotropic 2-plane becomes

$$
\left(\begin{array}{cccc}
\Gamma_{5} & \Gamma_{6} & \Gamma_{7} & \Gamma_{8} \\
0 & \Gamma_{5} & \Gamma_{6} & \Gamma_{7} \\
0 & 0 & \Gamma_{5} & \Gamma_{6}
\end{array}\right)\left(\begin{array}{c}
2 c_{1} c_{4}+2 c_{2} c_{3} \\
2 c_{2} c_{4}+c_{3}^{2} \\
2 c_{3} c_{4} \\
c_{4}^{2}
\end{array}\right)=\left(\begin{array}{l}
0 \\
0 \\
0 \\
0
\end{array}\right) .
$$

Since $\Gamma_{5}$ is non-zero, the above matrix has a 1-dimensional kernel. If $c_{4}$ is zero, then $c_{3}$ is also zero. In this case, any $X$ of the form $\left\langle c_{1} u_{1}+c_{2} u_{2}\right\rangle$ solves the above equation. However, for all such lines, $\operatorname{Span}\{X, T X\}$ contains the $T$-stable subspace $\left\langle u_{1}\right\rangle$, thereby forcing $X \notin L_{0}^{\prime f, T, *}\left(k^{s}\right)$. Note that if $c_{1} \neq 0$, then $\operatorname{Span}\{X, T X\}=\left\langle u_{1}, u_{2}\right\rangle$ and $X \in L_{2}^{\prime f, T, *}\left(k^{s}\right)$, violating condition (3.6), while every such $X$ still satisfies the weaker condition that it contains no non-zero $T$-stable subspace.

Now, given a non-zero $c_{4}$ obtained by taking a non-zero vector in the 1-dimensional kernel and taking a square root of the corresponding entry, there is a unique solution for $c_{1}, c_{2}$ and $c_{3}$. Therefore, there are $2=2^{1}$ choices for $X$ depending on which square root one chooses for $c_{4}$.

We have now completed the proof of Proposition 3.33.

Proof of Theorems 3.26 and 3.30. Applying Proposition 3.31 repeatedly gives a bijection

$$
L_{\left\{d_{1}, \ldots, d_{r+1}\right\}}^{\prime f, T, *}\left(k^{s}\right) \underset{\delta}{\sim} L_{\{0,0, \ldots, 0\}}^{\prime} \prod_{i}\left(x-\alpha_{i}\right)^{m_{i}-2 d_{i}, T^{\prime}, *}\left(k^{s}\right),
$$

and for any $X \in L_{\left\{d_{1}, \ldots, d_{r+1}\right\}}^{\prime f, T, *}\left(k^{s}\right)$,

$$
\left|\operatorname{Stab}_{J_{T}}(X)\right|=2^{a}\left|\operatorname{Stab}_{T^{\prime}}(\delta(X))\right| \text {. }
$$

This proves Theorem 3.30. The polynomial $g(x)=\prod_{i}\left(x-\alpha_{i}\right)^{m_{i}-2 d_{i}}$ has $r+1-a$ distinct roots, hence applying Proposition 3.33 to $g$ and then dividing by 2 to go from $\left|L^{\prime f, T, *}\right|$ to $\left|L^{\prime f, T}\right|$ completes the proof of Theorem 3.26. 


\section{PENCILS OF QUADRICS AND HYPERELLIPTIC CURVES}

\section{ACKNOWLEDGEMEnTS}

This paper is a revised version of part of my Harvard Ph.D. thesis written under the supervision of Benedict H. Gross. I am very grateful to him for introducing me to the subject and for many insightful discussions. I would also like to thank Manjul Bhargava, George Boxer, Brian Conrad, Anand Deopurkar, Joe Harris, Anand Patel, Bjorn Poonen, and Cheng-Chiang Tsai for valuable suggestions and conversations.

\section{REFERENCES}

BG13 M. Bhargava and B. H. Gross, The average size of the 2-Selmer group of Jacobians of hyperelliptic curves having a rational Weierstrass point, in Automorphic Representations and L-Functions, Tata Inst. Fundam. Res. Stud. Math., vol. 22 (Tata Inst. Fund. Res., Mumbai, 2013), 23-91.

BG14 Arithmetic invariant theory, in Symmetry: Representation Theory and its Applications, Progr. Math., vol. 257 (Birkhäuser/Springer, New York, 2014), 33-54; https://doi.org/10. 1007/978-1-4939-1590-3_3.

BGW17 M. Bhargava, B. H. Gross, and X. Wang, A positive proportion of locally soluble hyperelliptic curves over $\mathbb{Q}$ have no point over any odd degree extension, J. Amer. Math. Soc. 30 (2017), no. 2, 451-493; https://doi.org/10.1090/jams/863.

Bho90 U. N. Bhosle, Singular pencils of quadrics and compactified Jacobians of curves, Proc. Indian Acad. Sci. Math. Sci. 100 (1990), no. 2, 95-102; https://doi.org/10.1007/BF02880954.

Cas93 J.W.S. Cassels, Jacobian in genus 2, Math. Proc. Cambridge Philos. Soc. 114 (1993), no. 1, 1-8; https://doi.org/10.1017/S0305004100071346.

Don80 R. Donagi, Group law on the intersection of two quadrics, Ann. Scuola Norm. Sup. Pisa Cl. Sci. (4) 7 (1980), no. 2, 217-239; http://www . numdam.org/item?id=ASNSP_1980_4_7_2_217_0.

DR76 U. V. Desale and S. Ramanan, Classification of vector bundles of rank 2 on hyperelliptic curves, Invent. Math. 38 (1976), no. 2, 161-185; https://doi.org/10.1007/BF01408570.

Gau54 L. Gauthier, Footnote to a footnote of André Weil, Univ. e Politec. Torino. Rend. Sem. Mat. 14 (1954), 325-328.

Har92 J. Harris, Algebraic geometry. A first course, Grad. Texts in Math., vol. 133 (Springer-Verlag, New York, 1992); https://doi.org/10.1007/978-1-4757-2189-8.

Har77 R. Hartshorne, Algebraic geometry, Grad. Texts in Math., vol. 52 (Springer-Verlag, New York Heidelberg, 1977); https://doi.org/10.1007/978-1-4757-3849-0.

Mum70 D. Mumford, Abelian varieties, Tata Inst. Fundam. Res. Stud. Math., vol. 5 (Tata Inst. Fund. Res., Mumbai; Oxford Univ. Press, 1970).

PS97 B. Poonen and E. F. Schaefer, Explicit descent for Jacobians of cyclic covers of the projective line, J. reine angew. Math. 1997 (1997), no. 488, 141-188; https://doi.org/10.1515/crll. 1997.488 .141$.

Rei72 M. Reid, The complete intersection of two or more quadrics, Ph.D. thesis, Trinity College, Cambridge, 1972, available at https://homepages.warwick.ac.uk/ masda/3folds/qu.pdf.

SW18 A. Shankar and X. Wang, Rational points on hyperelliptic curves having a marked nonWeierstrass point, Compos. Math. 154 (2018), no. 1, 188-222; https://doi.org/10.1112/ S0010437X17007515.

Xiaoheng Wang x46wang@uwaterloo.ca

Department of Mathematics, Princeton University, Princeton, NJ 08544, USA

Current address: Pure mathematics department, University of Waterloo, Waterloo, Ontario N2L

3G1, Canada 